\title{
IONIZATION IN ATMOSPHERES OF BROWN DWARFS AND EXTRASOLAR PLANETS. V. ALFVÉN IONIZATION
}

\author{
C. R. Stark ${ }^{1}$, Ch. Helling ${ }^{1}$, D. A. Diver ${ }^{2}$, And P. B. Rimmer ${ }^{1}$ \\ ${ }^{1}$ SUPA, School of Physics and Astronomy, University of St Andrews, St Andrews, KY16 9SS, UK; craig.stark@st-andrews.ac.uk \\ ${ }^{2}$ SUPA, School of Physics and Astronomy, Kelvin Building, University of Glasgow, Glasgow, G12 8QQ, UK \\ Received 2013 February 19; accepted 2013 August 6; published 2013 September 19
}

\begin{abstract}
Observations of continuous radio and sporadic X-ray emission from low-mass objects suggest they harbor localized plasmas in their atmospheric environments. For low-mass objects, the degree of thermal ionization is insufficient to qualify the ionized component as a plasma, posing the question: what ionization processes can efficiently produce the required plasma that is the source of the radiation? We propose Alfvén ionization as a mechanism for producing localized pockets of ionized gas in the atmosphere, having sufficient degrees of ionization $\left(\geqslant 10^{-7}\right)$ that they constitute plasmas. We outline the criteria required for Alfvén ionization and demonstrate its applicability in the atmospheres of low-mass objects such as giant gas planets, brown dwarfs, and M dwarfs with both solar and sub-solar metallicities. We find that Alfvén ionization is most efficient at mid to low atmospheric pressures where a seed plasma is easier to magnetize and the pressure gradients needed to drive the required neutral flows are the smallest. For the model atmospheres considered, our results show that degrees of ionization of $10^{-6}-1$ can be obtained as a result of Alfvén ionization. Observable consequences include continuum bremsstrahlung emission, superimposed with spectral lines from the plasma ion species (e.g., $\mathrm{He}, \mathrm{Mg}, \mathrm{H}_{2}$, or $\mathrm{CO}$ lines). Forbidden lines are also expected from the metastable population. The presence of an atmospheric plasma opens the door to a multitude of plasma and chemical processes not yet considered in current atmospheric models. The occurrence of Alfvén ionization may also be applicable to other astrophysical environments such as protoplanetary disks.
\end{abstract}

Key words: brown dwarfs - magnetic fields - planets and satellites: atmospheres - plasmas - radiation mechanisms: general - stars: atmospheres

Online-only material: color figures

\section{INTRODUCTION}

Current atmospheric models of low-mass objects do not fully consider coherent plasma effects or collective plasma processes and how they fit into a holistic understanding of atmospheric phenomena such as cloud formation and evolution. Observations of continuous radio and sporadic X-ray emission from lowmass objects suggest that such objects harbor an atmospheric, magnetized plasma of some form. This fact suggests that atmospheres at the very least are composed of gas-plasma mixtures where dust (and, by extension, clouds) can grow, creating a dusty plasma (a plasma containing charged particulates of nanometer and micrometer size). Charged dust particles will be susceptible to inter-grain electrical discharges (Helling et al. 2011b, 2013) that will energize the ambient gas-plasma (MacLachlan et al. 2009, 2013; Li et al. 2009) and will participate in other collective plasma phenomena such as waves, flows, and instabilities. Therefore, it is key to our understanding of substellar atmospheres that the relevant plasma phenomena are incorporated into atmospheric models and their observational consequences quantified. Before all else, it is critical to identify the ionization processes that create and replenish the atmospheric plasma that may feed the extended envelope surrounding a low-mass object.

Low-mass objects with spectral type later than M7 (ultracool dwarfs) can be strong sources of radio emission that indicates the presence of atmospheric plasmas. Observations of the M9 dwarf TVLM 513-46546 at 4.88 and $8.44 \mathrm{GHz}$ characterize the radio emission as variable with a periodicity consistent with the estimated period of rotation $(\approx 2 \mathrm{hr}$; Hallinan et al. 2006). Follow-up observations identified additional periodic $(1.96 \mathrm{hr})$ bursts of $100 \%$ circularly polarized, coher- ent radio emission (Hallinan et al. 2007). Similar radio emission signatures have been detected from other ultracool dwarfs such as the T6.5 dwarf 2MASS J1047+21 (Route \& Wolszczan 2012), the M8.5 dwarf LSR J1835+3259, and the L3.5 dwarf 2MASS J00361617+1821104, with brightness temperatures indicating that emission must come from a coherent source, most likely an electron cyclotron maser instability (Hallinan et al. 2008).

The coherent nature of the emission allows the determination of the magnetic field strength; if an electron cyclotron maser is assumed, the radiation is predominately emitted at the harmonics of the electron cyclotron frequency. Modeling of the electron cyclotron maser emission mechanism has uncovered details of the expected electromagnetic signature arising when the maser is electron beam-driven, loss cone-driven (Yu et al. 2012), and driven by electrical currents resulting from an angular velocity shear (Nichols et al. 2012). Assuming an electron cyclotron maser emission mechanism, the required magnetic flux densities of the ultracool dwarfs must be approximately of the order of $1 \mathrm{kG}$, which is consistent with other observed radio emitting ultracool dwarfs (McLean et al. 2011, 2012; Osten et al. 2009; Antonova et al. 2007, 2008, 2013; Berger 2006; Berger et al. 2009).

Measurements of M-dwarf magnetic flux densities from the Zeeman effect, as a function of spectral type, show that $B \approx O(1 \mathrm{kG})$ (Shulyak et al. 2011; Reiners 2012). No direct observations have been made of exoplanetary magnetic fields but magnetic dynamo simulations suggest that magnetic flux densities of $\approx O(10 \mathrm{G})$ can be expected (Sánchez-Lavega 2004). This result is consistent with predicted magnetic field strengths of massive exoplanets by Christensen et al. (2009). These 
authors suggest a scaling law for inferring the magnetic field strength based on energy-flux considerations, consistent with the maximum magnetic fields found in rapidly rotating low-mass stars and planetary magnetic fields such as that of Jupiter. Transit observations of exoplanets may provide a method of deducing the magnitude of planetary magnetic fields by investigating the interaction between stellar winds and planetary magnetospheres (Vidotto et al. 2011a, 2011b).

Radio and X-ray observations suggest that plasmas are a potentially important component in the atmospheric environments of low-mass objects. A plasma is defined as a collection of charged particles, of sufficiently high number density that the Coulomb force is significant in determining its properties, yet sufficiently dilute that the nearest neighbor interaction is dominated not by binary collisions but instead by the collective electromagnetic influence of the many distant particles. Locally, the entirety of the gas does not need to be ionized for it to behave as a plasma; in fact, for an ionized gas to be considered a plasma, it only needs to be weakly ionized (i.e., partially ionized), provided the collective electromagnetic influence of the many distant particles is significant in determining the dynamics. A measure of the extent to which an ionized gas behaves like a plasma is the degree of ionization. The degree of ionization is defined as the ratio of the density of the charged species $n_{i}$ to that of the neutral species $n_{\mathrm{gas}}$, where $f_{e}=n_{i} /\left(n_{i}+n_{\mathrm{gas}}\right)=1$ is a completely ionized plasma and $f_{e} \approx 10^{-7}$ is a weakly ionized plasma (Diver 2013; Fridman 2008). If localized pockets of plasma can be created and sustained in an atmosphere, this situation would open the door to a multitude of plasma processes and chemistries not yet considered in current models of substellar and planetary objects.

Helling and co-workers have made progress in considering the effects of plasma phenomena in substellar atmospheres (Helling et al. 2011a, 2011b, 2013). Helling et al. (2011a) used DRIFTPHOENIX model atmospheres to investigate the effect of dustinduced collisional ionization. They found that ionization by turbulence-induced dust-dust collisions was the most efficient of the ionization processes considered but that the electron density produced was insufficient to significantly improve the degree of ionization. However, the resulting charged dust grains that compose the atmospheric clouds are susceptible to inter-grain electrical discharge events (Helling et al. 2011b).

In discharge events, the electric field permeating the intervening ambient gas exceeds a threshold value, resulting in the electrical breakdown of the gas. Helling et al. (2013) investigated the electrical breakdown conditions under which mineral clouds in substellar atmospheres undergo discharge events. Furthermore, the impact of these events on the local gas-phase chemistry, the effective temperature, and the primordial gas-phase metallicity was assessed. Needle-shaped grains can play an important role: the strong electric fields at the polar regions of the grain (Stark et al. 2006) are particularly sensitive to inter-grain discharges (MacLachlan et al. 2009, 2013; Li et al. 2009), enhancing the effect. An ensemble of such discharges, in analogy to laboratory microdischarges (Becker et al. 2006), will amplify the local degree of ionization to levels that the ionized gas constitutes a plasma (Helling et al. 2011b). If the ambient magnetic field permeating the atmosphere is sufficiently strong, the plasma will be magnetized, coupling the atmosphere to the magnetic field.

The question remains what ionization process can efficiently produce a plasma in the atmospheric environments of lowmass objects. The degree of thermal ionization is insufficient to qualify the ionized component as a plasma; however, inter-grain electrical discharge events (Helling et al. 2013) and cosmicray ionization processes (Rimmer \& Helling 2013) can occur that enhance the local degree of ionization. In this paper, we propose Alfvén ionization (also known as a critical ionization velocity phenomenon) as a simple mechanism for producing localized pockets of plasma in the atmospheres of low-mass objects. In Alfvén ionization, a constant stream of neutral gas impinges on a low-density magnetized plasma. The inflowing neutral atoms collide with and displace the plasma ions, leaving behind a significant charge imbalance that accelerates electrons to energies sufficient to ionize the local gas via electron-neutral impact ionization. Alfvén ionization requires an initial, lowdensity magnetized seed plasma and a neutral gas flow that reaches a critical threshold speed. As well as creating an ionized plasma, Alfvén ionization has significant implications for atmospheric chemistry.

This paper is structured as follows. Section 2 summarizes the non-phase-equilibrium atmosphere model (DRIFT-PHOENIX) of Helling and co-workers, of which a selected number of models will be used in the subsequent analysis. Section 3 introduces Alfvén ionization and discusses it in the context of planetary, brown dwarf, and M-dwarf atmospheres, defining the criteria of its applicability. The emission levels of ultracool dwarfs are similar to those of early type active M-dwarfs (Antonova et al. 2013) and so we are interested in the link connecting M-dwarfs, brown dwarfs, and giant gas planets. It is plausible that similar mechanisms for the production of the source plasma and the radio emission are at work. Section 4 quantifies the resulting degree of ionization expected from Alfvén ionization. Section 5 summarizes our findings and discusses the consequent scientific implications. Note that unless otherwise stated, the equations in this paper are given in SI units.

\section{ATMOSPHERE MODELS}

In this paper, we are interested in Alfvén ionization in the atmospheres of gas giant planets, brown dwarfs, and M-dwarfs. We utilize the DRIFT-PHOENIX atmosphere grid as described in Witte et al. (2011), which provides us with the necessary atmospheric input quantities. The thermodynamic structure ( $p_{\text {gas }}, T_{\text {gas }}$, and $\left.\rho_{\text {gas }}\right)$ of substellar atmospheres is complex and depends on a number of competing and corroborating factors. DRIFT-PHOENIX considers an atmosphere in hydrostatic and chemical equilibrium and utilizes mixing length and radiative transfer theories to consistently calculate the thermodynamic structure of the model atmospheres. We consider three representative sets of stellar parameters for each type of object: giant gas planets $\left(\log g=3.0, T_{\text {eff }}=1500 \mathrm{~K}\right.$; hereafter referred to as GP), brown dwarfs $\left(\log g=5.0, T_{\text {eff }}=1500 \mathrm{~K}\right.$; hereafter referred to as $\mathrm{BD})$, and $\mathrm{M}$-dwarfs $\left(\log g=4.0, T_{\text {eff }}=2700 \mathrm{~K}\right.$; hereafter referred to as $\mathrm{MD})$. We study solar $([\mathrm{M} / \mathrm{H}]=0.0)$ and sub-solar $([\mathrm{M} / \mathrm{H}]=-0.6)$ metallicities. Figure 1 shows the temperature-pressure profiles and the gas-phase mass density variation with atmospheric pressure for these respective model atmospheres calculated using DRIFT-PHOENIX. Additionally, as an example, Figure 2 shows the respective atomic and molecular number densities for selected species for a brown dwarf model atmosphere.

The DRIFT-PHOENIX cloud formation model of Helling and co-workers (Woitke \& Helling 2003; Helling et al. 2008a, 2008b) is fundamentally different from other contemporary cloud models. First and foremost, this model kinetically 

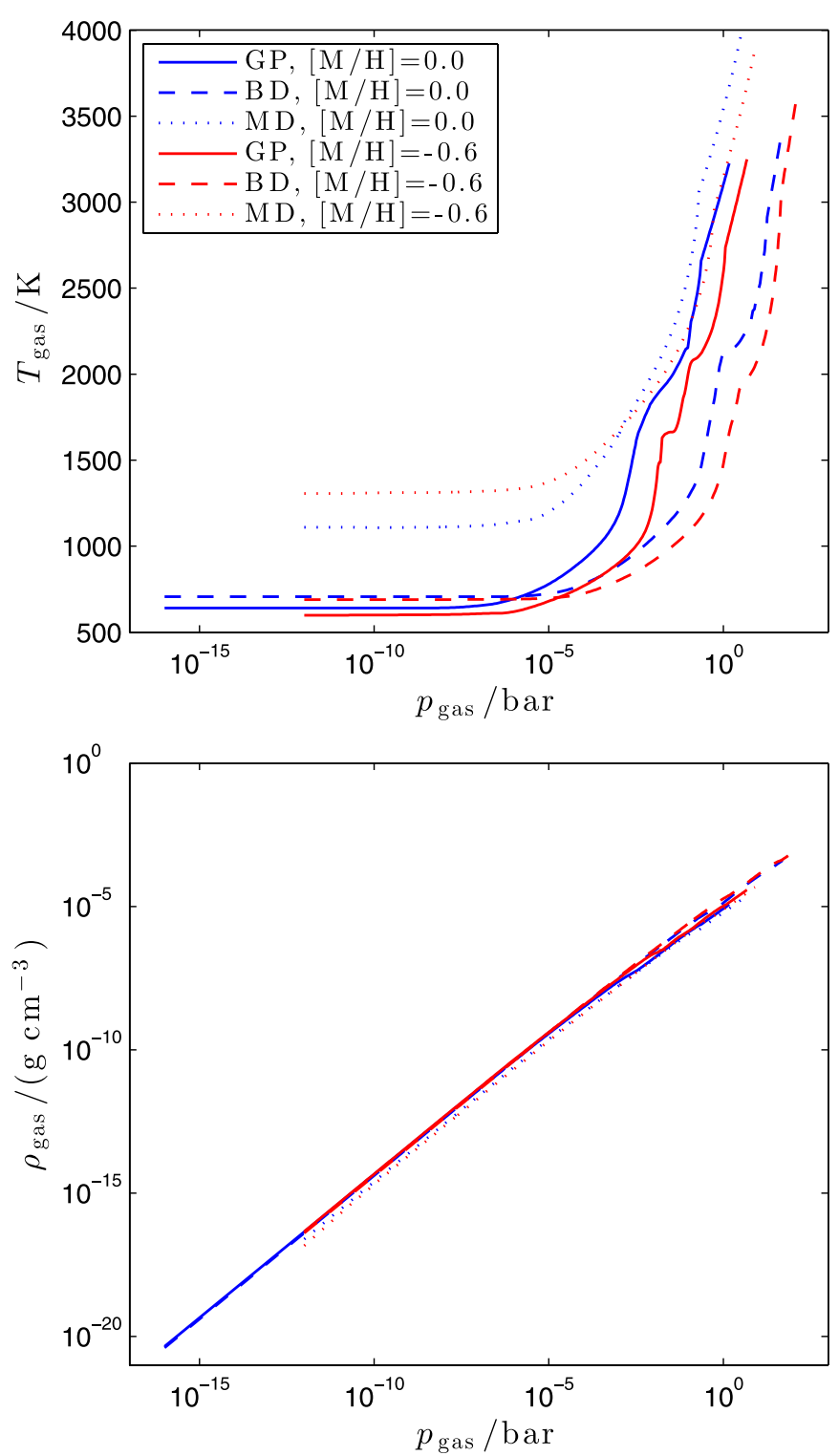

Figure 1. Atmospheric ( $p_{\text {gas }}, T_{\text {gas }}$; top panel) and ( $p_{\text {gas }}, \rho_{\text {gas }} ;$ bottom panel) diagrams for examples of gas giant planets $\left(\mathrm{GP}: \log g=3.0, T_{\text {eff }}=1500 \mathrm{~K}\right)$, brown dwarfs (BD: $\log g=5.0, T_{\text {eff }}=1500 \mathrm{~K}$ ), and M-dwarfs (MD: $\log g=4.0, T_{\text {eff }}=2700 \mathrm{~K}$ ). These results are obtained from DRIFT-PHOENIX simulations where solar elemental abundances $[\mathrm{M} / \mathrm{H}]=0.0$ (blue lines) and sub-solar abundances $[\mathrm{M} / \mathrm{H}]=-0.6$ (red lines) have been considered.

(A color version of this figure is available in the online journal.)

describes the formation of cloud particles as a phase-transition process by modeling seed formation, grain growth and evaporation, sedimentation in phase-non-equilibrium, element depletion, and the interaction of these collective processes. Second, unlike other approaches, the trajectory of an ensemble of dust grains is followed from the top of the atmosphere to the bottom. Additionally, Drift-PhoENIX includes the effect of dust formation on the ambient atmosphere in a feedback system that allows the calculation of self-consistent pressure-temperature profiles (Dehn 2007). In the cloud model, the gas phase is calculated assuming chemical equilibrium for 14 elements $(\mathrm{H}, \mathrm{He}$, $\mathrm{C}, \mathrm{N}, \mathrm{O}, \mathrm{Si}, \mathrm{Mg}, \mathrm{Al}, \mathrm{S}, \mathrm{Na}, \mathrm{K}, \mathrm{Ti}$, and $\mathrm{Ca}$ ) and 158 molecules. The model's initial elemental abundances are solar (or subsolar) and are changed by the dust formation process. The cloud model numerically solves a system of differential equations that describe a one-dimensional (1D) stationary dust formation pro-
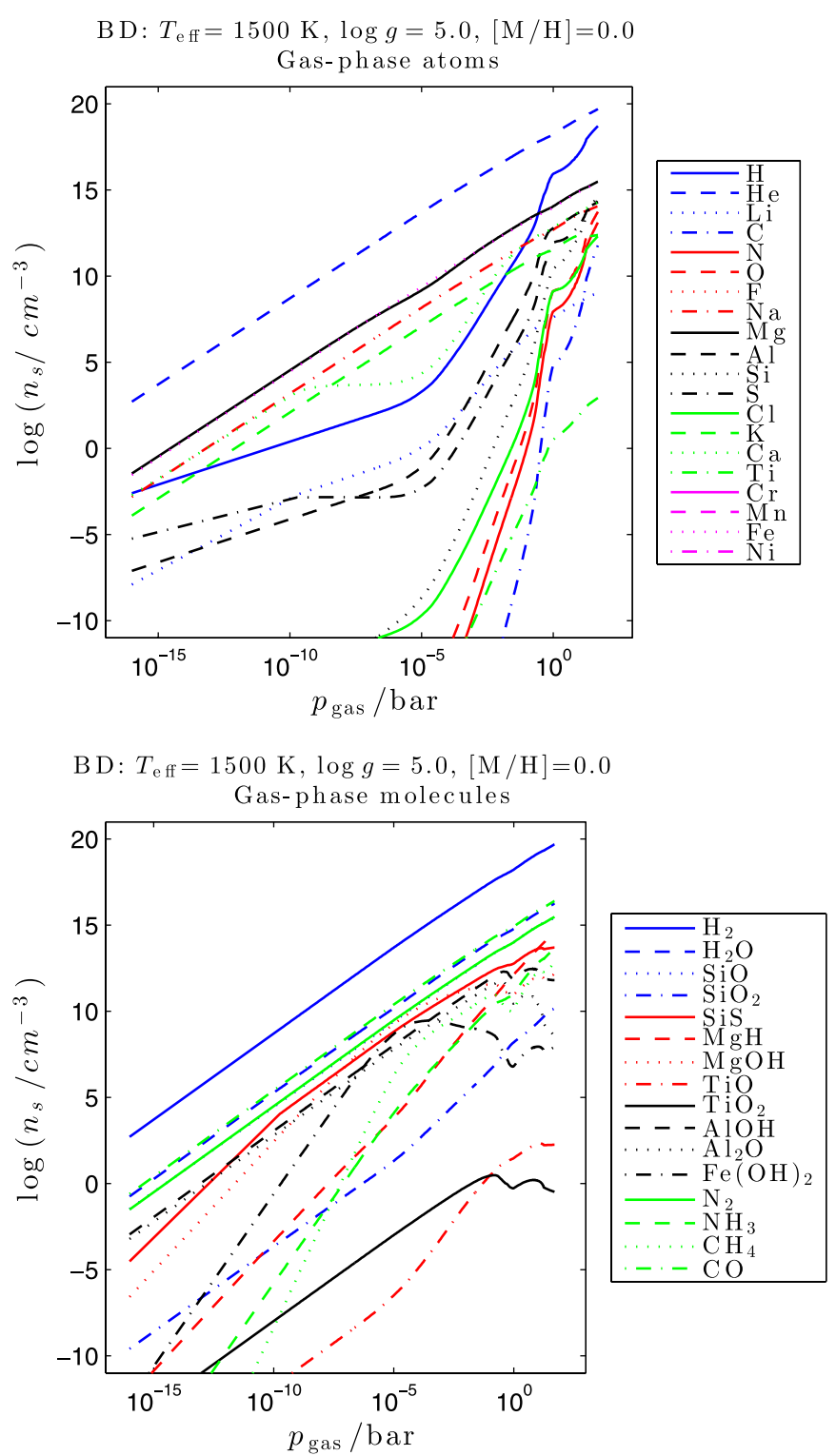

Figure 2. Atomic and molecular number densities $n_{s}$ for selected species $s$ within a brown dwarf (BD: $\left.T_{\text {eff }}=1500 \mathrm{~K}, \log g=5.0\right)$ model atmosphere assuming initially solar abundances $([\mathrm{M} / \mathrm{H}]=0.0)$. For clarity, the plots show a close-up of the most abundant species to emphasize their variation.

(A color version of this figure is available in the online journal.)

cess, where seeds form $\left(\mathrm{TiO}_{2}\right)$ from a highly supersaturated gas, grow to macroscopic particles of micrometer size, gravitationally settle, and eventually evaporate as the local temperature exceeds that required for thermal stability. Once the initial seed particles have formed, growth proceeds via surface chemical reactions that cause the formation of a grain mantle.

\section{ALFVÉN IONIZATION IN THE ATMOSPHERES OF LOW-MASS OBJECTS}

This section discusses Alfvén ionization in the context of substellar atmospheres. Section 3.1 introduces Alfvén ionization and discusses the process by which it can ionize an ambient gas. The criteria of its applicability are outlined in the context of planetary, brown dwarf, and M-dwarf atmospheres (Sections 3.2-3.6), making use of the DrIFT-PhoENIX model atmospheres discussed in Section 2. 
(1)
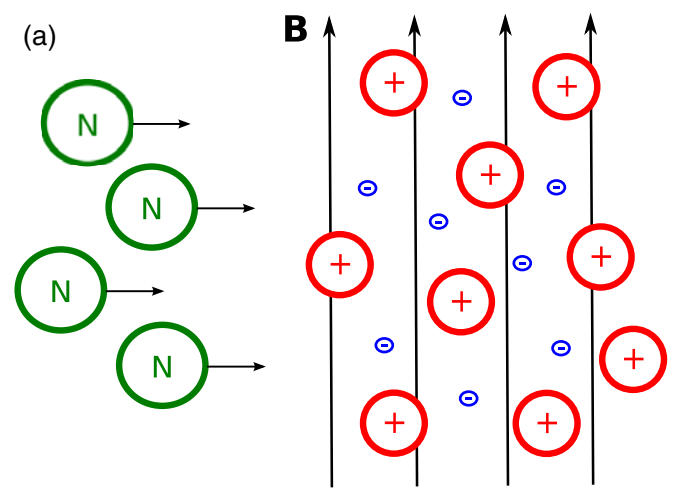

(b)

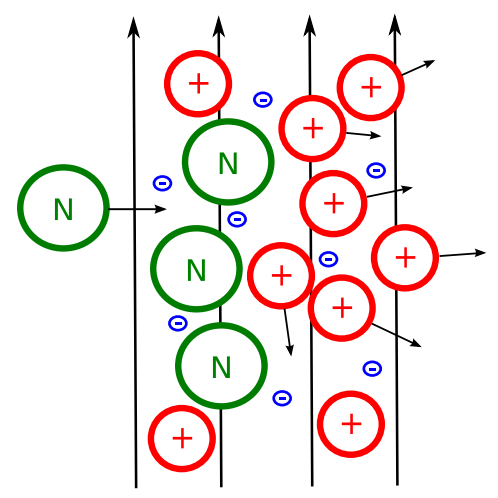

(c)

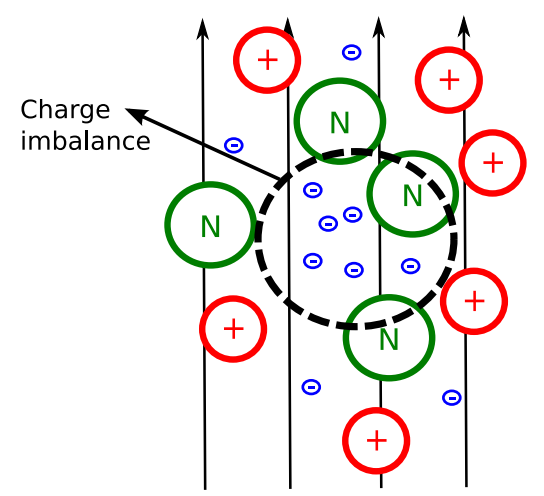

Figure 3. Alfvén ionization process: (a) a constant stream of neutral gas impinges on a low-density, localized, magnetized plasma; (b) the inflowing neutral atoms collide with and displace the plasma ions; (c) the displaced ions leave behind a significant charge imbalance that accelerates electrons to energies sufficient to ionize the local gas via electron-neutral impact ionization.

(A color version of this figure is available in the online journal.)

\subsection{Alfvén Ionization}

Consider a constant stream of neutral gas with a flow speed $v_{0}$ impinging on a low-density, stationary, magnetized seed plasma. The source of the seed plasma and the required magnetic flux density for the plasma to be sufficiently magnetized will be discussed in Sections 3.2 and 3.3. It is assumed that this seed plasma is localized by the ambient magnetic field and is of a similar chemical composition as the neutral gas. The inflowing neutral atoms collide with and displace the plasma ions from their equilibrium positions, producing a significant charge imbalance that cannot be rectified immediately due the restricted motion of the magnetized electrons (see Figure 3). The scattered ions are sent off in a Larmor orbit, leaving behind a localized pocket of electrons with a spatial length scale equal to the ion Larmor radius, which is defined as $R_{\mathrm{Li}}=v_{\perp i} / \omega_{\mathrm{ci}}$, where $v_{\perp i}$ is the speed of the ions perpendicular to the magnetic field and $\omega_{\mathrm{ci}}=q_{i} B / m_{i}$ is the ion cyclotron frequency of an ion of mass $m_{i}$ carrying a charge $q_{i}$ in a magnetic field. The resulting self-electrostatic field of the exposed electrons continues to grow until the potential difference inhibits further ionic displacement. At this point, the electrostatic potential energy is now equal to the maximum kinetic energy $m_{\text {gas }} v_{0}^{2 / 2}$ (where $m_{\text {gas }}$ is the mass of a neutral atom) of an ion as a result of a collision (Alfvén 1960). The resulting self-electric field (i.e., the self-repulsion of the electrons) accelerates the local electrons to an equivalent energy, ionizing the incoming neutral atoms that have an electron-impact ionization threshold, $e \phi_{I}$, that is less than $m_{\text {gas }} v_{0}^{2 / 2}$ (MacLachlan et al. 2009, 2013). Note that it is only the relative speed between the magnetized plasma and gas that is important; a moving magnetized plasma can encounter a stationary neutral component: the critical requirement is that the plasma is localized by the ambient magnetic field. The Alfvén ionization mechanism is easiest to visualize when there is an interface between the plasma and neutral species; however, the process can occur if the plasma is continuously distributed throughout a volume. The stochastic nature of collisions is sufficient to scramble the phase and timing of any ion trajectories, leaving the necessary required pockets of imbalanced electrons. In Alfvén ionization laboratory experiments conducted by Fahleson (1961), the plasma and neutral species were mixed and continuously distributed throughout the volume of the vessel. Many laboratory experiments have successfully exhibited Alfvén ionization (Brenning 1992; Danielsson 1973) and many have been performed in space (Newell 1985).

For Alfvén ionization, we require that (1) the seed plasma is strongly magnetized and (2) the neutral gas flow reaches a critical speed $v_{c}$. The critical speed is obtained by equating the kinetic energy of the flow to the electrostatic potential energy of an electron in an electric potential equal to the ionization potential of a neutral gas particle $\phi_{I}: m_{\mathrm{gas}} v_{c}^{2 / 2}=e \phi_{I}$ (Alfvén 1960). Therefore, the critical neutral gas flow speed is

$$
v_{c}=\left(\frac{2 e \phi_{I}}{m_{\text {gas }}}\right)^{1 / 2} \text {. }
$$

Tables 1 and 2 list the atomic weight, the first ionization potential, and the critical ionization speed, calculated using Equation (1), for selected atoms and molecules, typically found in the cool atmospheres of low-mass objects such as brown dwarfs, giant gas planets, and M-dwarfs. Atoms such as potassium $(\mathrm{K})$ and chromium $(\mathrm{Cr})$ have the smallest critical speeds $\left(v_{c}=4.63\right.$ and $5.01 \mathrm{~km} \mathrm{~s}^{-1}$, respectively), whereas hydrogen $(\mathrm{H})$ and helium $(\mathrm{He})$ have the largest speeds $\left(v_{c}=\right.$ 51.02 and $34.43 \mathrm{~km} \mathrm{~s}^{-1}$, respectively). For a given flow with kinetic energy $E_{k}=m_{\text {gas }} v_{0}^{2 / 2}$, neutrals with a smaller mass $m_{\text {gas }}$ require a greater flow speed $v_{0}$ to achieve equivalent kinetic energies. Energetically, condition (2) is similar to the condition required for the liberation of an electron from the surface of a dust grain (i.e., the work function) as a result of dust-dust or dust-gas collisions (Helling et al. 2011a).

Alfvén ionization requires that the local charge imbalance imposed by the gas flow be established on a timescale, $\tau_{s}$, shorter than that for electron transport to neutralize it, $\tau_{e}$ (Diver et al. 2005). Therefore, it is required that $\tau_{s} \ll \tau_{e}$, where $\tau_{s}$ is the setup time for collisions to create a charge imbalance and $\tau_{e}$ is the typical time for electron transport to eliminate the charge imbalance. Consider the case when the ions are magnetized: $\omega_{\mathrm{ci}} \gg v_{\mathrm{ni}}$, where $v_{\mathrm{ni}}$ is the ion-neutral collision 
Table 1

Table of Selected Atomic Gas-phase Species ${ }^{1}$ in Substellar Atmospheres

\begin{tabular}{lcccc}
\hline \hline Species & Symbol & $\begin{array}{c}\text { Atomic Weight } \\
\mathrm{u}\end{array}$ & $\begin{array}{c}\phi_{I} \\
(\mathrm{eV})\end{array}$ & $\begin{array}{c}v_{c} \\
\left(\mathrm{~km} \mathrm{~s}^{-1}\right)\end{array}$ \\
\hline Potassium & $\mathrm{K}$ & 39.1 & 12.13 & 4.22 \\
Chromium & $\mathrm{Cr}$ & 52 & 6.77 & 5.01 \\
Nickel & $\mathrm{Ni}$ & 58.69 & 7.64 & 5.01 \\
Manganese & $\mathrm{Mn}$ & 54.94 & 7.43 & 5.11 \\
Iron & $\mathrm{Fe}$ & 55.85 & 7.9 & 5.22 \\
Titanium & $\mathrm{Ti}$ & 47.88 & 6.83 & 5.25 \\
Calcium & $\mathrm{Ca}$ & 40.08 & 6.11 & 5.42 \\
Aluminum & $\mathrm{Al}$ & 26.98 & 5.99 & 6.54 \\
Sodium & $\mathrm{Na}$ & 22.99 & 5.14 & 6.57 \\
Silicon & $\mathrm{Si}$ & 28.09 & 8.15 & 7.48 \\
Magnesium & $\mathrm{Mg}$ & 24.3 & 7.65 & 7.79 \\
Sulfur & $\mathrm{S}$ & 32.07 & 10.4 & 7.91 \\
Chlorine & $\mathrm{Cl}$ & 35.45 & 13 & 8.41 \\
Lithium & $\mathrm{Li}$ & 6.94 & 5.39 & 12.24 \\
Oxygen & $\mathrm{O}$ & 16 & 13.6 & 12.81 \\
Fluorine & $\mathrm{F}$ & 19 & 17.4 & 13.29 \\
Carbon & $\mathrm{C}$ & 12.01 & 11.26 & 13.45 \\
Nitrogen & $\mathrm{N}$ & 14.01 & 14.5 & 14.13 \\
Neon & $\mathrm{Ne}$ & 20.18 & 21.6 & 14.37 \\
Helium & $\mathrm{He}$ & 4.0026 & 24.6 & 34.43 \\
Hydrogen & $\mathrm{H}$ & 1.0079 & 13.6 & 51.02 \\
\hline & & & &
\end{tabular}

Notes. The ionization potential $\phi_{I}$ and the critical ionization speed $v_{c}$ are calculated using Equation (1). The table is ordered with respect to the magnitude of $v_{c}$, from smallest to largest.

Reference: (1) Diver et al. 2005.

frequency. When the ions are scattered by neutral collisions, they participate in a Larmor orbit with a frequency $\omega_{\text {ci }}$. As long as the electrons cannot rectify the charge imbalance during this timescale (i.e., before the ions return to their initial positions and neutralize the charge imbalance), Alfvén ionization will occur. In this case, the critical timescale to create a charge imbalance is the ion cyclotron frequency, $\tau_{s} \approx \omega_{\mathrm{ci}}^{-1}$. However, the ions do not necessarily need to be magnetized for the Alfvén ionization process; for example, in Fahleson (1961), the ions are initially unmagnetized. If unmagnetized, the ions undergo many neutral collisions in an ion cyclotron period and so are not likely to return as compensating charge to their original equilibrium positions. This situation presents a significant relaxation of our more demanding requirement since we do not formally require the ions to be magnetized, only the electrons; hence, the criteria for Alfvén ionization is more easily fulfilled. In this case, the critical timescale would be the rate at which ions are displaced due to neutral collisions: $\tau_{s} \approx v_{\mathrm{ni}}^{-1}$. There have been numerous theoretical and numerical studies of the Alfvén ionization process that have investigated the ion-neutral beam interaction and the resulting electron energization (McBride et al. 1972; Machida \& Goertz 1986; Person et al. 1990; McNeil et al. 1990).

For gas mixtures, the critical ionization speed $v_{c}$ depends on the relative abundance and critical speeds of the individual participating species. In experiments conducted using twospecies gas mixtures, $v_{c}$ is intermediate between those of the two individual species. The behavior of such multi-species mixtures is not well understood and at best can be described only by empirical relations (Lai 2001). For more complex systems, where there is a greater number of participating species (such as in the atmospheres of low-mass objects), we will assume that the individual species are treated in isolation.
Table 2

Table of Selected Molecular Gas-phase Species in Substellar Atmospheres

\begin{tabular}{lccc}
\hline \hline Species & $\begin{array}{c}\text { Atomic Weight } \\
\mathrm{u}\end{array}$ & $\begin{array}{c}\phi_{I} \\
(\mathrm{eV})\end{array}$ & $\begin{array}{c}v_{c} \\
\left(\mathrm{~km} \mathrm{~s}^{-1}\right)\end{array}$ \\
\hline $\mathrm{TiO}$ & 63.87 & 6.819 & 4.53 \\
$\mathrm{TiO}_{2}$ & 79.87 & 9.5 & 4.79 \\
$\mathrm{MgOH}$ & 41.31 & 7.5 & 5.91 \\
$\mathrm{SiO}$ & 44.08 & 11.3 & 7.03 \\
$\mathrm{MgH}$ & 25.31 & 6.9 & 7.25 \\
$\mathrm{SiO}$ & 60.08 & 18 & 7.6 \\
$\mathrm{CO}$ & 28.01 & 14.0 & 9.7 \\
$\mathrm{~N}_{2}$ & 28.01 & 15.6 & 10.4 \\
$\mathrm{NH}_{3}$ & 17.04 & 10.02 & 10.65 \\
$\mathrm{H}_{2} \mathrm{O}$ & 18.02 & 12.6 & 11.6 \\
$\mathrm{CH}_{4}$ & 16.04 & 12.61 & 12.31 \\
$\mathrm{H}_{2}$ & 2.01 & 15.60 & 38.5 \\
\hline
\end{tabular}

Notes. The ionization potential $\phi_{I}$ and the critical ionization speed $v_{c}$ are calculated using Equation (1). The table is ordered with respect to the magnitude of $v_{c}$, from smallest to largest.

\subsection{Seed Plasma}

To satisfy condition (1) for Alfvén ionization, we require an initial low-density, magnetized seed plasma to initiate the ionization process. In this section, we will compare the length scale over which a plasma can effectively screen a charge imbalance with the length scale over which the Alfvén ionization process can create a charge imbalance. This calculation will determine the required threshold electron number density of the seed plasma needed to trigger Alfvén ionization.

As part of the Afvén ionization process, the inflowing neutrals scatter the ambient ions, thereby exposing a localized pocket of electrons, the self-repulsion of which accelerates the electrons to sufficient energies that collisionally ionize the surrounding neutrals. Due to the elastic collisions with the neutrals, the scattered ions are given a speed (equal to the neutral flow speed $v_{0}$ ) perpendicular to the ambient magnetic field and are sent off in a Larmor orbit, leaving behind the magnetized electrons. The localized pocket of electrons has a spatial length scale equal to the ion Larmor radius, $R_{\mathrm{Li}}$.

For a plasma, the Debye length is defined as

$$
\lambda_{D}=\left(\frac{\epsilon_{0} k_{B} T_{e}}{n_{e} e^{2}}\right)^{1 / 2},
$$

where $T_{e}$ is the electron temperature and $n_{e}$ is the ambient electron number density of the plasma. The Debye length defines the spatial length scale at which exposed charge is screened by the plasma; at scales greater than the Debye length, the plasma is considered neutral and experiences no effects due to the charge imbalance. At scales less than the Debye length, the charge imbalance is not screened and the influence of the resulting electric field is significant. Therefore, to allow a localized pocket of electrons (a charge imbalance) of length scale $R_{\mathrm{Li}}$ to be created, it is required that the Debye length be much smaller than the ion Larmor radius:

$$
\begin{gathered}
\lambda_{D} \ll R_{\mathrm{Li}}, \\
\left(\frac{\epsilon_{0} k_{B} T_{e}}{n_{e} e^{2}}\right)^{1 / 2} \ll \frac{m_{i} v_{\perp i}}{e B} .
\end{gathered}
$$

Rearranging for $n_{e}$, the criterion can be recast in terms of the ambient electron number density of the seed plasma required to 


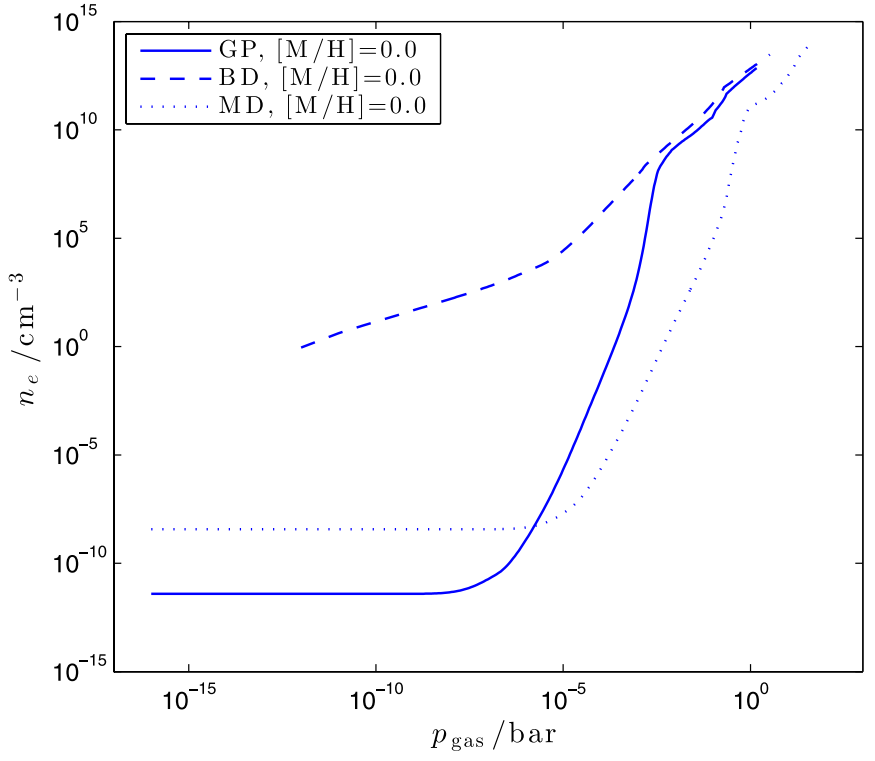

Figure 4. Ambient electron number density $n_{e}$ as a function of atmospheric pressure $p_{\text {gas }}$. The electron number density results from thermal ionization in the atmosphere of a gas giant planet (GP: $\log g=3.0, T_{\text {eff }}=1500 \mathrm{~K}$ ), brown dwarf (BD: $\left.\log g=5.0, T_{\text {eff }}=1500 \mathrm{~K}\right)$, and M-dwarf $(\mathrm{MD}: \log g=4.0$, $\left.T_{\text {eff }}=2700 \mathrm{~K}\right)$ for a solar abundance $([\mathrm{M} / \mathrm{H}]=0.0)$.

(A color version of this figure is available in the online journal.)

initiate Alfvén ionization:

$$
n_{e}^{\text {seed }} \gg \frac{\epsilon_{0} k_{B} T_{e} B^{2}}{m_{i}^{2} v_{\perp i}^{2}} .
$$

To calculate what threshold electron number density $n_{e}^{\text {seed }}$ is required, we set $v_{\perp i}=v_{0} \approx 1 \mathrm{~km} \mathrm{~s}^{-1}$ and approximate $m_{i} \approx 10^{-27} \mathrm{~kg}$. Although some of the neutral species have a critical speed $O\left(10 \mathrm{~km} \mathrm{~s}^{-1}\right)$, setting $v_{\perp i}=1 \mathrm{~km} \mathrm{~s}^{-1}$ gives an upper limit to $n_{e}^{\text {seed }}$. Therefore, the number density of the magnetized seed plasma must satisfy

$$
n_{e}^{\text {seed }} \gtrsim 10^{2} T_{e} B^{2}\left[\mathrm{~cm}^{-3}\right] .
$$

This expression for $n_{e}^{\text {seed }}$ is dependent upon the electron temperature $T_{e}$ and the magnetic flux density $B$. Plasma electron temperatures can range from $T_{e} \approx 10^{2}$ to $10^{6} \mathrm{~K}$ (Fridman 2008), where laboratory microplasmas (Becker et al. 2006) and terrestrial lightning strikes can produce $T_{e} \approx 10^{4} \mathrm{~K}(\approx 1 \mathrm{eV})$. In the atmospheres of low-mass objects, thermal ionization produces an electron population with $T_{e} \approx 10^{2}-10^{3} \mathrm{~K}$, in thermal equilibrium with the ambient atmospheric gas. Typical average, global (large-scale) magnetic flux densities for GPs are estimated to be of the order of $\approx 10 \mathrm{G}$; BDs and MDs have much greater flux densities on the order of $1 \mathrm{kG}$ (Donati \& Landstreet 2009; Reiners 2012; Christensen et al. 2009; Sánchez-Lavega 2004; Shulyak et al. 2011). For $B \approx 10-10^{3} \mathrm{G}$ and $T_{e} \approx 10^{2}-10^{6} \mathrm{~K}$, the resulting range in the seed electron number density is $n_{e}^{\text {seed }} \gtrsim 10^{6}-10^{16} \mathrm{~cm}^{-3}$. For a typical GP atmosphere with $T_{e} \approx O(1000 \mathrm{~K})$ and $B \approx O(10 \mathrm{G})$, the threshold seed electron number density is $n_{e}^{\text {seed }} \gtrsim 10^{7} \mathrm{~cm}^{-3}$. For $\mathrm{BD}$ and $\mathrm{MD}$ atmospheres with a similar order of magnitude electron temperature $\left(T_{e} \approx O(1000 \mathrm{~K})\right)$ and $B \approx O(1 \mathrm{kG})$, the threshold seed density is $n_{e}^{\text {seed }} \gtrsim 10^{11} \mathrm{~cm}^{-3}$.

In the atmospheres of low-mass objects, ambient electron number densities from thermal ionization are expected to reach up to $n_{e} \approx 10^{12} \mathrm{~cm}^{-3}$ deep in the atmosphere $\left(p_{\text {gas }} \approx 1\right.$ bar; see

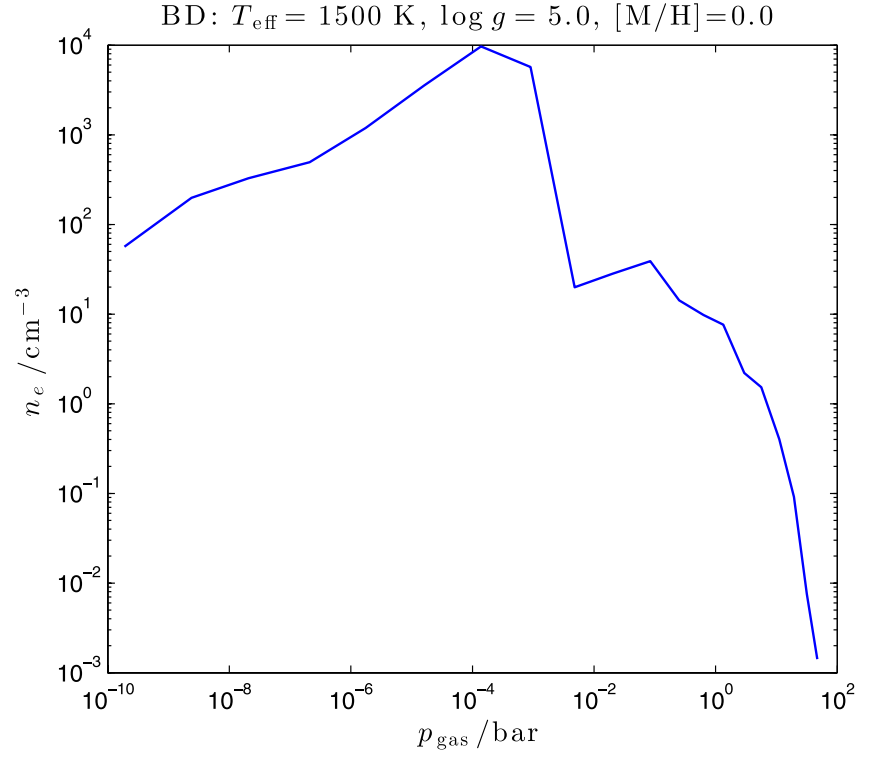

Figure 5. Electron number density $n_{e}$ resulting from the cosmic-ray bombardment of a brown dwarf atmosphere (BD: $\log g=5.0, T_{\text {eff }}=1500 \mathrm{~K}$; $[\mathrm{M} / \mathrm{H}]=0.0)$.

(A color version of this figure is available in the online journal.)

Figure 4). However, at higher altitudes, the atmospheric pressure and temperature decrease and the gas becomes insufficiently energetic to maintain the same level of thermal ionization, and hence $n_{e}$ decreases. As an example, for a $\mathrm{BD}$ atmosphere with $[\mathrm{M} / \mathrm{H}]=0.0, n_{e}$ falls from $\approx 10^{12} \mathrm{~cm}^{-3}$ (at $p_{\text {gas }} \approx 1$ bar) to $\approx 10 \mathrm{~cm}^{-3}$ (at $p_{\text {gas }} \approx 10^{-12}$ bar).

Figure 4 shows the electron number density as a function of atmospheric pressure due to thermal ionization alone (resulting from Saha equilibrium). Although $n_{e}$ falls to low values in the upper regions of the atmosphere, we expect that the electron number density will be enhanced via other ionizing processes such as cosmic-ray ionization and atmospheric inter-grain electrical discharges (there are other processes too), locally yielding an electron number density that exceeds the threshold value required for the seed plasma. Clouds in low-mass objects are susceptible to local discharge events (lightning) that can significantly enhance the local electron density (Helling et al. 2011b, 2013). For example, observations of terrestrial lightning strikes show an electron number density $n_{e} \approx 10^{17} \mathrm{~cm}^{-3}$ in a single discharge event (Uman \& Orville 1964; Guo et al. 2009; Chang et al. 2010). Such lightning strikes are analogous to gas discharges used in terrestrial laboratory plasmas, such as microdischarge devices, where characteristic plasma number densities can reach $\approx 10^{16} \mathrm{~cm}^{-3}$ (Becker et al. 2006).

Additionally, cosmic ray bombardment of the atmosphere will significantly increase $n_{e}$ via impact ionization of the initially neutral atmosphere. Figure 5 shows the resulting electron number density $n_{e}$ from the bombardment of a brown dwarf atmosphere $([\mathrm{M} / \mathrm{H}]=0.0)$ by cosmic rays. The plot shows that high in the atmosphere $\left(p_{\text {gas }} \approx 10^{-4}\right.$ bar $)$, cosmic ray ionization processes can increase the ambient electron number density to $n_{e} \approx 10^{4} \mathrm{~cm}^{-3}$. For more details regarding the cosmic ray calculation, see Appendix A.

Taking into account these additional contributions to the electron number density, it is reasonable to presume that the seed plasma required for Alfvén ionization is achievable in the atmospheres of low-mass objects such as M-dwarfs, brown dwarfs, and gas planets. 


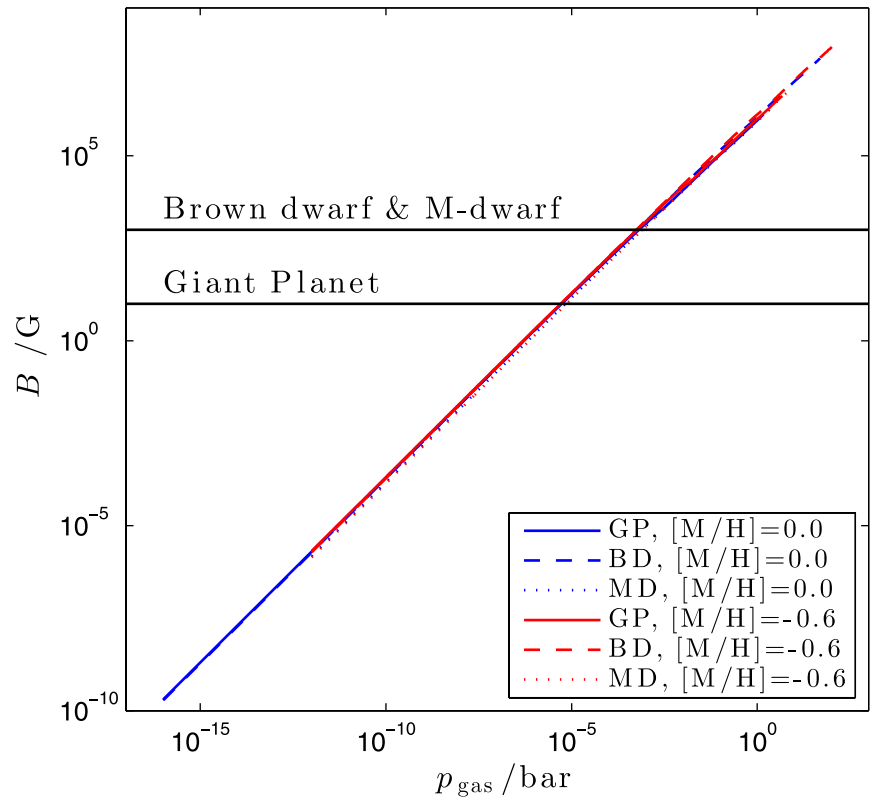

Figure 6. Magnetized plasma criterion (Equation (9)) in the atmospheres of low-mass objects: gas giant planets (GP: $\log g=3.0, T_{\text {eff }}=1500 \mathrm{~K}$; solid line), brown dwarfs (BD: $\log g=5.0, T_{\text {eff }}=1500 \mathrm{~K}$; dashed line), and M-dwarfs (MD: $\log g=4.0, T_{\text {eff }}=2700 \mathrm{~K}$; dotted line) for both solar abundance $([\mathrm{M} / \mathrm{H}]=0.0$, blue lines $)$ and low metallicity $([\mathrm{M} / \mathrm{H}]=-0.6$, red lines). The plot shows the required minimum magnetic flux density $B$ for the plasma to qualify as magnetized. The horizontal black lines show the typical average, global (large-scale) magnetic flux densities for GPs, BDs, and MDs.

(A color version of this figure is available in the online journal.)

\subsection{Magnetized Seed Plasma}

Furthermore, to satisfy condition (1) for Alfvén ionization, the background seed plasma must be magnetized. Since Alfvén ionization works when the electrons are magnetized, whether or not the ions are too, ensuring that the electrons are magnetized is sufficient. The criterion for magnetized electrons is $\omega_{\text {ce }} \gg v_{\text {coll }}$, where $\omega_{\mathrm{ce}}=e B / m_{e}$ is the electron cyclotron frequency, $m_{e}$ is the mass of an electron, and $v_{\text {coll }}$ is the electron-neutral collision frequency. This expression simply states that for the plasma dynamics to be significantly influenced by a magnetic field, a charged particle must complete on average at least one gyration before participating in a collision. The criterion for magnetized electrons can be rewritten as

$$
\begin{aligned}
\frac{e B}{m_{e}} & \gg n_{\mathrm{gas}}\langle\sigma v\rangle, \\
B & \gg \frac{m_{e} n_{\mathrm{gas}}\langle\sigma v\rangle}{e}, \\
& \approx \frac{m_{e} n_{\mathrm{gas}} \pi r_{0}^{2}}{e}\left(\frac{k_{B} T_{e}}{m_{e}}\right)^{1 / 2},
\end{aligned}
$$

where the approximations $\langle\sigma v\rangle \approx \pi r_{0}^{2}\langle v\rangle$ and $\langle v\rangle=$ $\left(k_{B} T_{e} / m_{e}\right)^{1 / 2}$ have been made; $r_{0}$ is the atomic radius. DRIFTPhoENIX simulations provide the atmospheric pressure and temperature structure ( $\left.p_{\text {gas }}, T_{\text {gas }}\right)$ for GP, BD, and MD atmospheres (Figures 1 and 2). The required local magnetic flux density $B$ needed to establish a magnetized plasma for Alfvén ionization can be calculated for each of these atmospheres.

Figure 6 shows the criteria for a magnetized plasma (Equation (9)) in the atmospheres of low-mass objects: GP,
$\mathrm{BD}$, and $\mathrm{MD}$ atmospheres for both $[\mathrm{M} / \mathrm{H}]=0.0$ and $[\mathrm{M} / \mathrm{H}]=$ -0.6 . In these calculations, the atomic radius has been approximated as $r_{0} \approx 10^{-8} \mathrm{~cm}$ and $T_{e} \approx T_{\text {gas }}$. For a typical atmosphere such as for a brown dwarf $([\mathrm{M} / \mathrm{H}]=0.0$; see Figures 1 and 2$)$ with $p_{\text {gas }} \approx 10^{-10}$ bar, $n_{\text {gas }} \approx 10^{8} \mathrm{~cm}^{-3}$, and $T_{e} \approx 700 \mathrm{~K}$, the magnetized plasma criterion is $B \gtrsim 10^{-4} \mathrm{G}$. This result means that for the plasma to be considered magnetized, the ambient magnetic flux density must be at least $10^{-4} \mathrm{G}$.

Figure 6 shows that very little discriminates between the criteria for the various atmospheric models. Only deep in the respective atmospheres, where the gas number density is high, is a significant magnetic flux density required to satisfy the criterion. As the gas density increases, the plasma electrons are more likely to collide with the neutrals inhibiting their gyration and so disrupt the influence of the ambient magnetic field. High in the atmosphere ( $p_{\text {gas }} \approx 10^{-12}$ bar), $B \gtrsim 10^{-6} \mathrm{G}$ is required, whereas deep in the atmosphere, the criterion is much more severe $\left(B \gtrsim 10^{6} \mathrm{G}\right)$. However, over a large atmosphere pressure range $\left(10^{-15} \lesssim p_{\text {gas }} \lesssim 10^{-2}\right.$ bar $)$, the magnetized plasma criterion is easily achievable and the minimum local magnetic flux density required is $\lesssim 10^{4} \mathrm{G}$. Note that GP and $\mathrm{BD}$ atmospheric models for $[\mathrm{M} / \mathrm{H}]=0.0$ extend to lower atmospheric pressures ( $p_{\text {gas }} \approx 10^{-16}$ bar) where the magnetized criterion is $B \gtrsim 10^{-10} \mathrm{G}$; additionally, BD models for both $[\mathrm{M} / \mathrm{H}]=0.0$ and -0.6 can achieve $p_{\text {gas }} \approx 10$ bar deep in the atmosphere where $B \gtrsim 10^{8} \mathrm{G}$.

GPs have large-scale magnetic flux densities of the order of $10 \mathrm{G}$; whereas, BDs and MDs are of the order of $1 \mathrm{kG}$ (Donati \& Landstreet 2009; Reiners 2012; Christensen et al. 2009; Sánchez-Lavega 2004; Shulyak et al. 2011). However, note that stronger, localized (small-scale) magnetic flux densities may be likely; for example, the Sun's dipole magnetic flux density (a large-scale field) has a magnitude $\approx O(10 \mathrm{G})$, but small-scale fields, e.g., a sunspot, can have magnitudes $\approx O(1 \mathrm{kG})$ (Priest 1985; Borrero et al. 2008). Although the low-mass objects we consider are physically different from the Sun, the principle still applies that local fluctuations in the mean field can lead to favorable enhancements in field strength of the order required for optimum performance in our description. Therefore, it can be expected that the seed plasma is sufficiently magnetized in the majority of the atmosphere and can be considered a magnetized plasma. However, such local enhancements of the magnetic flux density may not be readily achievable for GPs since the source of magnetic amplification, expected in BDs and MDs, may be absent. In addition, the magnetic flux density of GPs is expected to be much weaker in strength $(10 \mathrm{G})$ compared with that of BDs and MDs $(1 \mathrm{kG})$. As a result, Alfvén ionization is harder to achieve in GPs. However, the nature of exoplanetary magnetic fields is still open for discussion and further investigation; e.g., the enhancement of the magnetic field strength in exoplanets can be achieved through the formation of magnetodisks (Khodachenko et al. 2012).

We have assumed that the seed plasma has had sufficient time to come into thermal equilibrium with the local gas and that it can be approximated to have a similar temperature, $T_{e} \approx T_{\mathrm{gas}}$. In the DRIFT-PhoENIX simulations, the electrons liberated by thermal ionization are in thermal equilibrium with the ambient gas and so this approximation is valid; however, this situation may not be the case for plasma electrons produced from other processes such as gas discharges. In reality, the plasma electrons need not be in thermal equilibrium with the ions or neutrals (a non-thermal plasma) and their temperature may be lower or much higher than these species (Fridman 


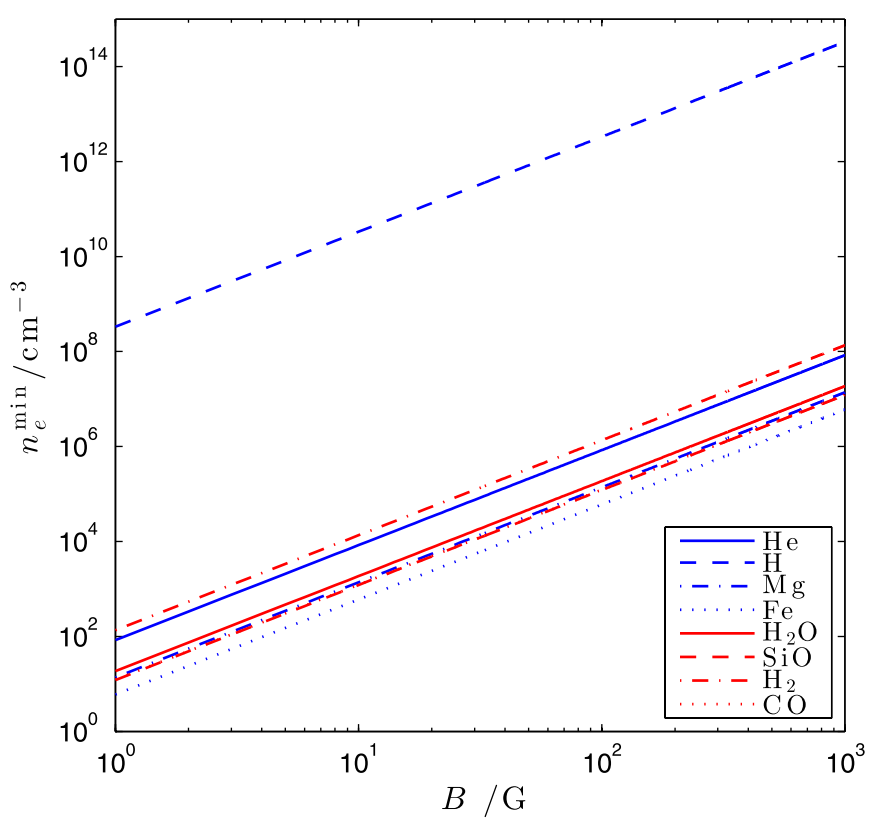

Figure 7. Minimum electron number density $n_{e}^{\min }$ (Equation (12)) required to produce the self-field for electron-impact ionization to occur for selected gas phase species. The plot shows $n_{e}^{\min }$ as a function of the magnetic flux density $B$ for selected gas phase species. The bottom plot shows how $n_{e}^{\min }$ varies with the atmospheric gas $\left(p_{\text {gas }}, T_{\text {gas }}\right)$ for hydrogen and a constant magnetic flux density $B=100 \mathrm{G}$.

(A color version of this figure is available in the online journal.)

2008). For example, laboratory plasma discharges can have electron temperatures $T_{e} \approx 1-10 \mathrm{eV}$ (equivalent to $10^{4}-10^{5} \mathrm{~K}$; Diver 2013; Fridman 2008) and can vary between 0.01-1 eV (equivalent to $10^{2}-10^{4} \mathrm{~K}$ ) in the Earth's ionosphere (Fridman 2008). The effect of having a greater electron temperature would be to increase the minimum threshold magnetic flux density for the atmospheric plasma to be considered magnetized; for example, if $T_{e} \approx 1 \mathrm{eV}$ (or a higher temperature, $T_{e} \approx 100 \mathrm{eV}$ ), the minimum magnetic flux density required would increase by a factor $\approx 3(\approx 30)$ relative to the case $T_{e}=1000 \mathrm{~K}$ $\left(T_{e} \approx 0.1 \mathrm{eV}\right)$. This effect is model independent since we are discussing an additional population of electrons that arise from ionizing processes other than those calculated using the DRIFTPhOENIX atmospheric model.

\subsection{Minimum Electron Number Density for Alfvén Ionization}

In Alfvén ionization, a local pocket of unscreened electrons (a charge imbalance) is produced by the inflowing neutrals. The self-electric field of the electrons (their collective electrostatic self-repulsion) accelerates the electrons to energies that can ionize the surrounding neutrals via electron-impact ionization. In order for the electrons to ionize the neutrals, the number density of the unscreened electron pocket must be great enough to facilitate the acceleration of the electrons to the appropriate ionization energy. The pocket of electrons will have a net charge $Q$ and a corresponding electrostatic potential, with a length scale equal to the ion Larmor radius $R_{\mathrm{Li}}$. For these electrons to successfully ionize a neutral gas with an ionization potential $\phi_{I}$, the electrostatic potential of the localized pocket must equal $\phi_{I}$ :

$$
\phi_{I}=\frac{Q}{4 \pi \epsilon_{0} R_{\mathrm{Li}}} .
$$

Furthermore, the volume of the charge pocket is $\approx R_{\mathrm{Li}}^{3}$, hence $Q \approx e n_{e} R_{\mathrm{Li}}^{3}$; therefore,

$$
\phi_{I}=\frac{n_{e} e R_{\mathrm{Li}}^{2}}{4 \pi \epsilon_{0}} .
$$

As such, the minimum electron number density $n_{e}^{\min }$ of the charge imbalance required to produce the self-field for electronimpact ionization can be calculated:

$$
n_{e}^{\min } \geqslant \frac{4 \pi \epsilon_{0} \phi_{I} Z_{i}^{2} e B^{2}}{m_{i}^{2} v_{\perp i}^{2}},
$$

where $R_{\mathrm{Li}}=m_{i} v_{\perp i} /\left(q_{i} B\right)$ is the Larmor radius, $q_{i}=Z_{i} e$, and $Z_{i}$ is the ion charge number. Assuming that $Z_{i}=1$, $v_{\perp i}=v_{c}=\left(2 e \phi_{I} / m_{\mathrm{gas}}\right)^{1 / 2}$, and $m_{i} \approx m_{\mathrm{gas}}$, then

$$
n_{e}^{\min } \geqslant \frac{2 \pi \epsilon_{0} B^{2}}{m_{\mathrm{gas}}} .
$$

For a GP with a magnetic flux density of $10 \mathrm{G}$ and an $\mathrm{H}_{2}$ dominated gas, the minimum electron number density needed to produce the required self-field is $n_{e}^{\min } \gtrsim 10^{4} \mathrm{~cm}^{-3}$. In the atmosphere of a $\mathrm{BD}$ or $\mathrm{MD}$ where magnetic flux densities can be $\approx 1 \mathrm{kG}, n_{e}^{\min } \approx 10^{8} \mathrm{~cm}^{-3}$ for an $\mathrm{H}_{2}$-dominated gas.

Figure 7 shows the minimum electron number density, $n_{e}^{\min }$ as a function of magnetic flux density $B$ for a range of gas phase species. In this approximation, the species' dependence on $n_{e}^{\min }$ is through the term $m_{i}^{-1}$, where species with a small mass require a larger $n_{e}^{\min }$; hence, hydrogen requires a greater $n_{e}^{\min }$ than iron. For example, the minimum electron number density for a hydrogen (iron) dominated plasma when $B \approx 10^{2} \mathrm{G}$ is $n_{e}^{\min } \geqslant 10^{12} \mathrm{~cm}^{-3}\left(\geqslant 10^{5} \mathrm{~cm}^{-3}\right)$. As $B$ is increased, $R_{\mathrm{Li}}$ decreases as the ions are forced to take part in smaller orbits. As a result, so that the same electric potential $\left(\phi_{I}\right)$ can be maintained, $n_{e}^{\min }$ must increase since $\phi_{I} \propto n_{e}^{\min } R_{\mathrm{Li}}^{2}$.

\subsection{Metastable States of Gas-phase Species}

In general, if the electron-neutral impact energy is insufficient to attain the ionization energy of the target particle, the energy may be sufficient such that the bound electrons are excited into higher electronic states and subsequently return to a lower energy state by radiating a photon. The timescale of such an electronic transition is usually of the order of $10^{-8} \mathrm{~s}$ (Bransden \& Joachin 2000); however, certain neutral atoms and molecules have excited states that persist for longer timescales (see Table 3). Such atoms and molecules struggle to decay to lower energy states because they cannot relax via dipole radiation and so must do so via higher-order transitions that are less efficient. Such metastable states can have lifetimes of the order of seconds (Dunning \& Hulet 1996) and can have a big impact on the distribution of energy within substellar atmospheres. Metastable transitions have been observed in the ejecta of $\eta$ Carinae (Zethson et al. 2001; Hartman et al. 2005)

Metastable states can play a crucial role in Alfvén ionization, where metastable species can interact with another energetic electron, this time imparting enough energy to ionize the excited species. The advantage is that the ionizing electron does not need to transfer energy equivalent to the ionization energy of the species, only the difference in energy between the metastable state and the ionization energy. Therefore, a lower Alfvén ionization critical speed is required. This fact means 
Table 3

Selected Metastable States That may Play a Significant Role in Alfvén Ionization and the Chemistry Occurring in Substellar Atmospheres ${ }^{1}$

\begin{tabular}{lcccc}
\hline \hline Species & State & $\begin{array}{c}\text { Energy } \\
(\mathrm{eV})\end{array}$ & $\begin{array}{c}\text { Approximate Lifetime } \\
(\mathrm{s})\end{array}$ & $\begin{array}{c}v_{c} \\
\left(\mathrm{~km} \mathrm{~s}^{-1}\right)\end{array}$ \\
\hline $\mathrm{H}$ & $2 S_{1 / 2}$ & 10.19 & 0.15 & 44.14 \\
\hline $\mathrm{He}$ & $2^{3} S_{1}$ & 19.82 & $7.9 \times 10^{3}$ & 30.9 \\
& $2^{1} S_{0}$ & 20.61 & $2 \times 10^{-2}$ & 31.5 \\
\hline $\mathrm{H}_{2}$ & $C^{3} \Pi_{u}$ & 11.75 & $1 \times 10^{-3}$ & 33.56 \\
& ${ }^{1} \Sigma_{g}(v=1)$ & 0.52 & $4 \times 10^{6}$ & 7.06 \\
\hline $\mathrm{N}_{2}$ & $A^{3} \Sigma_{u}^{+}$ & 6.17 & 2.7 & 6.52 \\
& $A^{1} \Sigma_{u}^{-}$ & 8.40 & 0.7 & 7.6 \\
& $A^{3} \Pi_{u}^{+}$ & 8.55 & $1.7 \times 10^{-4}$ & 7.67 \\
& $E^{3} \Sigma_{g}^{+}$ & 11.87 & $2 \times 10^{-4}$ & 9.04 \\
\hline
\end{tabular}

Reference: (1) Dunning \& Hulet 1996.

that for metastable species such as helium or $\mathrm{H}_{2}$, the species can be ionized in two stages without needing to reach the total critical velocity $\left(v_{c}=34.43 \mathrm{~km} \mathrm{~s}^{-1}\right.$ and $v_{c}=38.5 \mathrm{~km} \mathrm{~s}^{-1}$, respectively); a lower speed is sufficient. Hence, metastable species are easier to ionize. Table 3 gives a selection of metastable states appropriate to the atmospheres considered here.

The required critical speed for some metastable states is still quite hard to achieve; for example, the metastable state $2^{3} S_{1}$ of He requires $v_{c}=30.9 \mathrm{~km} \mathrm{~s}^{-1}$ (see Table 3 ). However, the critical speed $v_{c}$ is a bulk fluid commodity, averaged over a particle energy distribution function; in reality, only a high energy tail of the distribution function is required to attain these metastable states and the bulk, average speed for the remaining energy to fully ionize the neutral. Hence, it may be enough to have the mean fluid speed much smaller than $v_{c}$ since the energetic tail of the underlying particle distribution function might be populated by enough particles to excite the metastable state initially.

Furthermore, ionization can also occur through the collision of metastable neutrals $\left(X^{*}\right)$ with neutral particles of another species $(Y)$ if the excited metastable state has an energy equal to or greater than the ionization potential of the neutral atom. This process is known as the Penning ionization effect (Fridman 2008):

$$
X^{*}+Y \rightarrow X+Y^{+}+e^{-} \text {. }
$$

As an example, consider metastable $\mathrm{He}(19.82 \mathrm{eV})$ and neutral $\mathrm{Na}\left(\phi_{I}=12.13 \mathrm{eV}\right)$. Should these species collide, the latter will be ionized and the former will return to its ground state. An observable consequence of the Penning effect will be the presence of forbidden lines in observed spectra that are not present in the non-ionizing case.

It is useful to quantify what fraction of an atmosphere is in a metastable state. Assuming the system is in thermodynamic equilibrium with temperature $T$ and that it can be described by a canonical ensemble, the probability that the system is in state $E_{n}$ is given by

$$
p_{n}=\frac{g_{n} \exp \left(-E_{n} /\left(k_{B} T\right)\right)}{\sum_{i} g_{i} \exp \left(-E_{i} /\left(k_{B} T\right)\right)},
$$

where $g_{j}$ is the degeneracy factor of the microstate $j$ and the sum is over all energy levels. Therefore, the probability ratio of the system being in the ground state (gs) relative to the metastable state $(\mathrm{ms}), p_{\mathrm{ms}} / p_{\mathrm{gs}}$, can be calculated. Hence, the relative number density $n_{\mathrm{ms}}$ of helium that exists in the

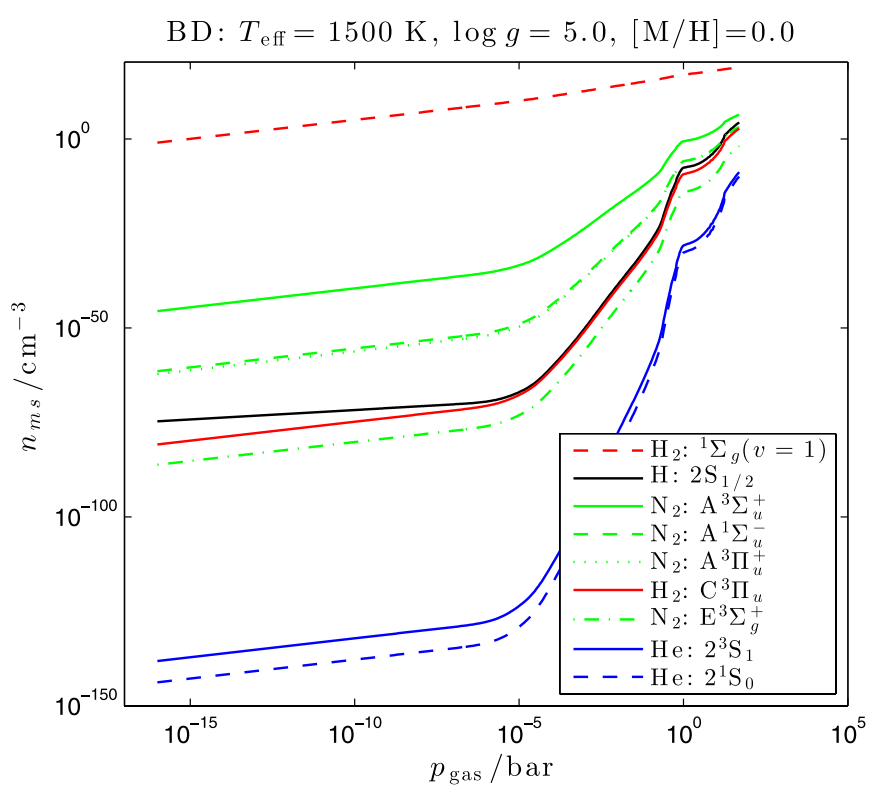

Figure 8. Number density of selected metastable states $n_{\mathrm{ms}}$ for a brown dwarf (BD: $\log g=5.0, T_{\mathrm{eff}}=1500 \mathrm{~K} ;[\mathrm{M} / \mathrm{H}]=0.0$ ), calculated using Equation (16). Information regarding the metastable states can be found in Table 3 .

(A color version of this figure is available in the online journal.)

metastable state with energy $E_{\mathrm{ms}}$ and degeneracy factor $g_{\mathrm{ms}}$ is given by

$$
n_{\mathrm{ms}}=n_{\mathrm{gs}} \frac{g_{\mathrm{ms}}}{g_{\mathrm{gs}}} \exp \left(-\frac{\Delta E}{k_{B} T}\right),
$$

where $n_{\mathrm{gs}}$ is the number density of the species in the ground state, $g_{\mathrm{gs}}$ is the degeneracy factor of the ground state, and $\Delta E$ is the energy difference between the ground state $E_{\mathrm{gs}}$ and the metastable state $E_{\mathrm{ms}}$. Figure 8 shows the number density of metastables in a brown dwarf atmosphere $([\mathrm{M} / \mathrm{H}]=0.0)$ as a function of atmospheric pressure and temperature $\left(p_{\mathrm{gas}}, T_{\mathrm{gas}}\right)$, for selected metastable species (listed in Table 3 ). We consider the metastable states of $\mathrm{H}, \mathrm{He}$, and $\mathrm{H}_{2}$ primarily because they are the hardest to ionize (i.e., they have the largest critical speed) via Alfvén ionization. $\mathrm{He}$ and $\mathrm{H}_{2}$ are of further interest since they have the largest number density in the atmosphere; $\mathrm{N}_{2}$ is interesting from a thermal equilibrium perspective since it is a very stable molecule. Thermally, there is insufficient energy in the atmosphere to excite the selected species into their metastable states to produce a significant number density $n_{\mathrm{ms}}$; metastable $\mathrm{He}$ is totally negligible. The metastable states of molecular nitrogen $\left(\mathrm{N}_{2}\right)$ yield higher number densities $\left(n_{\mathrm{ms}} \approx\right.$ $\left.10^{-15} \mathrm{~cm}^{-3}\right)$ but not significantly so. One metastable state of $\mathrm{H}_{2}$ $\left({ }^{1} \Sigma_{g}(v=1)\right)$ yields a modest $n_{\mathrm{ms}} \approx 10^{10} \mathrm{~cm}^{-3}$ at $p_{\text {gas }} \approx 1$ bar; however, the energy of the microstate is very small $(0.52 \mathrm{eV})$ so that its contribution to the overall Alfvén ionization process is insignificant.

In the atmospheric models considered here, the ambient atmosphere does not harbor a significant population of metastable species produced from thermal excitation. For an atmosphere to have a significant population of metastables, the atmospheric temperature and/or the density of the ground state species (corresponding to the metastable species) would have to be greater. In the model atmosphere results considered here, excitation to metastable states would need to occur via an alternative process such as gas discharges, cosmic-ray interactions, or even as part of the Alfvén ionization mechanism where bombardment from neutral flow drives the excitation. 
For example, simulations of electron avalanches (the precursor to a full gas discharge) in a $1 \mathrm{~mm}$ gap of atmospheric nitrogen have shown that the metastable density can reach $\approx 10^{12} \mathrm{~cm}^{-3}$ (MacLachlan et al. 2013). Furthermore, if in a localized volume of a $\mathrm{BD},[\mathrm{M} / \mathrm{H}]=0.0$ model atmosphere (for example at an atmospheric pressure of $\approx 10^{-10}$ bar), Alfvén ionization excited all the helium from its ground state to its first metastable state, the metastable density would be $\approx 10^{10} \mathrm{~cm}^{-3}$ (see Figure 2).

\subsection{Atmospheric Flows and the Critical Speed $v_{c}$}

For Alfvén ionization, condition (2) must be satisfied, i.e., the neutral gas flow speed must reach the critical flow speed $v_{c}$ which, for most gas species of interest, is approximately $O\left(1-10 \mathrm{~km} \mathrm{~s}^{-1}\right)$. It is therefore natural, in the context of neutral gas atmospheres around planetary and substellar objects, to look at dynamic meteorology and atmospheric flows to ascertain if such flow speeds are plausible.

In the absence of significant ionization, the atmospheric seed plasma is a minority species relative to the neutral species if only thermal ionization is considered. Therefore, the dynamics of the atmospheric gas-plasma mixture will be dominated by those of the neutral gas. Note that this statement does not mean that the plasma component is not magnetized (or coupled to the magnetic field), but rather that the dynamical effect of the plasma is a second order effect relative to the neutral species. We are proposing Alfvén ionization as a process to significantly enhance the degree of ionization and boost the plasma population, allowing the subsequent use of continuum models (such as magnetohydrodynamics, cold or warm plasma theory, etc). With this statement in mind, it is reasonable to omit plasma behavior for now and focus on neutral gas flows as a starting point (which are unaffected by the magnetic field) to see if we can create a significant plasma via Alfvén ionization. However, if an atmospheric plasma can be established (via Alfvén ionization) and sustained, the effect of the magnetic field on flows will be important and should be incorporated into the governing equations of motion.

There has been significant progress in meteorological studies of extrasolar planetary atmospheres and, by analogy, brown dwarfs, with sophisticated numerical simulations of global atmospheric circulatory systems (Showman \& Guillot 2002; Showman et al. 2008, 2009; Menou \& Rauscher 2009; Rauscher \& Menou 2010; Cooper \& Showman 2005; Dobbs-Dixon \& Lin 2008; Dobbs-Dixon et al. 2010; Dobbs-Dxion et al. 2012; Lewis et al. 2010; Heng et al. 2011).

The atmospheric dynamics of hot Jupiters are of particular interest because of their high temperatures and short orbital periods. Three-dimensional simulations of the atmospheric circulation of the hot Jupiters HD 209458b and HD 189744b by Showman \& Guillot (2002) and Showman et al. (2008, 2009) globally solve the primitive equations governing the atmospheric gas dynamics and compare the infrared spectra and light curves predicted by the simulations to the observational data. Their models produce equatorial jets with flow speeds of $3-4 \mathrm{~km} \mathrm{~s}^{-1}$, which are of the order of the magnitude required to trigger Alfvén ionization. Menou \& Rauscher (2009) solve the primitive equations using a pseudospectral solver for a shallow three-dimensional hot Jupiter model of HD 209458b and find that in the barotropic regime, zonal jets develop that obtain speeds up to $10 \mathrm{~km} \mathrm{~s}^{-1}$ (Rauscher \& Menou 2010). This result is consistent with the meteorological simulations of Cooper \& Showman (2005). Dobbs-Dixon and co-workers have incorporated radiative transfer and viscous effects into their hydrodynamical simulations, yielding gas flows of $\approx 5 \mathrm{~km} \mathrm{~s}^{-1}$ (Dobbs-Dixon \& Lin 2008; Dobbs-Dixon et al. 2010). Heng (2012) investigated supersonic flows (flows that approach or exceed $\approx 1 \mathrm{~km} \mathrm{~s}^{-1}$ ) in irradiated exoplanetary atmospheres and found that shocks form on the dayside hemisphere upstream of the substellar point due to the enhanced temperatures that develop there.

Modeling of the atmospheric circulation of the hot Neptune GJ436b has provided insights into the effects of metallicity on the dynamical and radiative behavior of extrasolar giant planetary atmospheres. As the atmospheric metallicity increases from solar levels to 50 times solar, the maximum zonal wind speeds reached increase from $\approx 1.3 \mathrm{~km} \mathrm{~s}^{-1}$ to over $2 \mathrm{~km} \mathrm{~s}^{-1}$, suggesting that zonal wind speeds increase with increasing metallicity (Lewis et al. 2010).

The meteorology of solar system planets has also been extensively studied; tracked cloud features on Uranus (Hammel et al. 2001; Sromovsky \& Fry 2005) and Saturn (Porco et al. $2005)$ imply wind speeds of $\approx O\left(0.1 \mathrm{~km} \mathrm{~s}^{-1}\right)$, which are too slow for Alfvén ionization to be a feasible process in these atmospheres. However, this result refers only to the observable parts of these atmospheres.

Our aim here is not to emulate such seminal meteorological models, but instead to examine simple atmospheric balanced flows to construct a compelling and feasible argument for the attainment of the required $v_{c}$ for Alfvén ionization. Balanced flows are an idealized view of actual atmospheric motion, but often give a good approximation of actual flows and yield a good estimation of the characteristic flow speeds. On a fundamental level, meteorological phenomena are driven by pressure variations and rotation and so it is possible to cast $v_{c}$ in terms of the required pressure gradient to drive neutral gas flows for a given rotation frequency. The underlying physical mechanisms that drive the pressure variations will vary depending on the specific object in question. For example, for planetary bodies, differential heating caused by illumination from a stellar companion will be the primary driver. For substellar objects, however, magnetic activity and/or resistive heating may be more significant. We will investigate this idea in a forthcoming paper. For simplicity, in the following treatment we do not prescribe a specific process but instead look at the physical ramifications of the atmospheric pressure variations produced by such processes. Here, we look at three standard balanced flows: geostrophic, cyclostrophic, and gradient flows. For a more detailed discussion regarding these balanced flows, see Appendix B.

\subsubsection{Geostrophic Flows}

Consider a local Cartesian tangent plane $(x, y)$ with latitude $\phi$ on the surface of a spherical body, rotating with angular speed $\omega$. In a rotating reference frame, a fluid element of atmospheric gas experiences the pressure gradient force, the Coriolis effect, the centrifugal effect, and gravity. Geostrophic wind balance results from the balance between the Coriolis effect and the pressure gradient force:

$$
u_{y}=\frac{1}{f \rho_{\mathrm{gas}}} \frac{\partial p_{\mathrm{gas}}}{\partial x},
$$

where $f=2 \omega \sin \phi$ is the Coriolis parameter characterizing the significance of the Coriolis effect as a function of position on the surface. For Alfvén ionization to be a feasible process, we require gas flows of $\left|u_{y}\right| \approx O\left(10 \mathrm{~km} \mathrm{~s}^{-1}\right)$. This requirement places the following constraint on the pressure gradient required 
to drive such flows:

$$
\left|\frac{\partial p_{\text {gas }}}{\partial x}\right| \geqslant f \rho_{\text {gas }}\left[\text { bar }^{-1}\right],
$$

where $f$ and $\rho_{\text {gas }}$ are in $\mathrm{rad} \mathrm{s}^{-1}$ and $\mathrm{g} \mathrm{cm}^{-3}$, respectively. Note that the magnitude of the pressure gradient is proportional to the gas mass density $\rho_{\text {gas }}$. Geostrophic flows are a good approximation to actual wind flows outside of the tropics (Holton 2004).

\subsubsection{Cyclostrophic Flows}

Transforming from Cartesian to cylindrical coordinates $(r, \theta, z)$ in a locally rotating non-inertial frame (such as a storm system or cyclone), an atmospheric fluid element experiences local centrifugal and local Coriolis effects in addition to the global effects of the rotating body. Cyclostrophic flows are characterized by the force balance between the pressure gradient force and the local centrifugal effect:

$$
u_{\theta}=\left(\frac{r}{\rho_{\mathrm{gas}}} \frac{\partial p_{\mathrm{gas}}}{\partial r}\right)^{1 / 2}
$$

For Alfvén ionization $\left(\left|u_{\theta}\right| \approx O\left(10 \mathrm{~km} \mathrm{~s}^{-1}\right)\right)$, this balance is achievable if the pressure gradient obeys

$$
\left|\frac{\partial p_{\text {gas }}}{\partial r}\right| \geqslant 1 \times 10^{6} \frac{\rho_{\text {gas }}}{r}\left[\text { bar cm }^{-1}\right],
$$

where $r$ and $\rho_{\text {gas }}$ are in $\mathrm{cm}$ and $\mathrm{g} \mathrm{cm}^{-3}$, respectively. As in the geostrophic case, the required pressure gradient is proportional to the gas mass density $\rho_{\text {gas }}$ and is additionally inversely proportional to the radius of the weather system, $r$. Therefore, smaller (larger) storm systems will yield a more severer (more lenient) pressure gradient criterion. Note that in the subsequent analysis, we will consider a weather system with circular motion of radius $r \approx 1000 \mathrm{~km}$. This size is comparable to the white oval storm systems observed on Jupiter (Beebe 1997; Choi et al. 2010). The Great Red Spot storm system has a spatial extent of the order of $O\left(10^{4} \mathrm{~km}\right)$ (Beebe 1997) and so larger storm systems are possible. Cyclostrophic flows are characteristic of weather systems such as tornadoes (Holton 2004).

In comparison, geostrophic and cyclostrophic flows only differ by a multiplicative factor, depending on the angular frequency and the radius of the weather system:

$$
\frac{\left|\partial p_{\text {gas }} / \partial x\right|}{\left|\partial p_{\text {gas }} / \partial r\right|}=1 \times 10^{-6} f r
$$

\subsubsection{Gradient Flows}

Remaining in cylindrical coordinates, gradient flows are characterized by the balance between the global Coriolis effect, the pressure gradient force, and the local centrifugal effect:

$$
u_{\theta}=-\frac{1}{2} r f \pm \frac{1}{2} r\left(f^{2}+\frac{4}{r \rho_{\mathrm{gas}}} \frac{\partial p_{\mathrm{gas}}}{\partial r}\right)^{1 / 2}
$$

For flow speeds $\left|u_{\theta}\right| \approx O\left(10 \mathrm{~km} \mathrm{~s}^{-1}\right)$, this balance will occur if the pressure gradient obeys

$$
\begin{aligned}
\left|\frac{\partial p_{\text {gas }}}{\partial r}\right| \geqslant & 2.5 \times 10^{-7} r \rho_{\text {gas }} \\
& \times\left[\frac{1}{r^{2}}\left(2 \times 10^{6}-r f\right)^{2}-f^{2}\right]\left[\text { bar cm }^{-1}\right],
\end{aligned}
$$

where $r$ and $\rho_{\text {gas }}$ are in $\mathrm{cm}$ and $\mathrm{g} \mathrm{cm}^{-3}$, respectively. Note that the pressure gradient criterion is directly proportional to the gas mass density $\rho_{\text {gas }}$; for a given value of $r$ and $f$, it is equal to a constant times $\rho_{\text {gas }}$. With increasing rotation period, $f \rightarrow 0$ and gradient flows reduce to cyclostrophic flows. In the following analysis, we will consider a low-pressure system $\left(\partial p_{\text {gas }} / \partial r>0\right.$ and $u_{\theta}>0$ ) with circular motion of radius $r \approx 1000 \mathrm{~km}$.

In conclusion, for the balanced flows considered here, the pressure gradient criteria are proportional to $\rho_{\text {gas }}$, for a given rotation period and/or weather system radius (characterized by the Coriolis parameter $f$ and radius $r$, respectively). Therefore, depending on the chosen values of $f$ and $r$, the relative performance between the three flows can be different. For example, for a storm system with a small (large) radius $r$, cyclostrophic flows could produce a pressure gradient criterion that has a greater (smaller) magnitude than that of geostrophic flows.

\subsubsection{Flows in Substellar Atmospheres}

Consider a low-mass object rotating with an angular frequency $\omega=3.5 \times 10^{-4} \mathrm{rad} \mathrm{s}^{-1}$ (i.e., a rotation period of $T=5 \mathrm{hr}$ ), Therefore, the Coriolis parameter is $f \approx 4.9 \times$ $10^{-4} \mathrm{rad} \mathrm{s}^{-1}$ for $\phi=\pi / 4$ (mid-latitudes). Exoplanets are expected to have rotation periods ranging from 1 to 4 days for hot Jupiters, 10-20 hr for Jupiter-like planets, and 2 to $3 \mathrm{hr}$ for fast-rotators approaching centrifugal breakup (Sánchez-Lavega $2001,2004)$. Brown dwarfs are considered to be very rapid rotators with average rotation periods $\leqslant 1$ day, with a lower limit of $\approx 2-4 \mathrm{hr}$ (Scholz \& Eislöffel 2005; Joergens et al. 2003; Zapatero Osorio et al. 2006). M-dwarfs have been observed to have rotation periods ranging from $\approx 7$ to $150 \mathrm{hr}$ (Reiners et al. 2012). Radio activity (and hence the presence of an atmosphere plasma) appears to be correlated with fast rotators, justifying our choice of rotation period $T=5 \mathrm{hr}$, which is close to the centrifugal breakup of the low-mass objects considered here.

Figures 9-11 show the pressure gradient criteria for geostrophic (Equation (18)), cyclostrophic (Equation (20)), and gradient flows (Equation (23)), respectively. These figures shows how these flows vary throughout the atmosphere $\left(p_{\text {gas }}, T_{\text {gas }}\right)$ for all three groups of low-mass object considered here, with solar elemental abundances and low metallicity. These plots show the minimum required pressure gradient needed to drive flows at the critical speed $\left(\left|u_{\theta}\right| \approx O\left(10 \mathrm{~km} \mathrm{~s}^{-1}\right)\right)$ needed for Alfvén ionization to occur. DRIFT-PHOENIX models obey 1D hydrostatic equilibrium, hence no horizontal wind velocity profiles can be provided.

For all the balanced flows considered here, the pressure gradient criteria are equal to a multiplicative factor times the gas mass density $\rho_{\text {gas }}$ (for a given $f$ and $r$ ). Therefore, for a single model atmosphere, the different balanced flows will have the same functional form and will only differ by a multiplicative factor. For example, for a particular model atmosphere, the gradient and cyclostrophic criteria for $f \approx 4.9 \times 10^{-4} \mathrm{rad} \mathrm{s}^{-1}$ and $r=1000 \mathrm{~km}$ are indistinguishable (Figures 10 and 11). Moreover, since the different model atmospheres considered here have similar $\left(p_{\text {gas }}, \rho_{\text {gas }}\right)$ profiles, the respective pressure gradient criteria are similar. However, GP and BD models for $[\mathrm{M} / \mathrm{H}]=0.0$ extend to lower mass densities and pressures in comparison to the other models considered here (Figure 1) and so in these atmospheres where the pressure is low, it is easier to fulfill the criteria for the balanced flows discussed here.

In general, the pressure gradient is directly proportional to the gas density $\rho_{\text {gas }}$ and so Figures $9-11$ show that at high altitudes $\left(p_{\text {gas }} \approx 10^{-16}-10^{-12}\right.$ bar) where the atmosphere is 


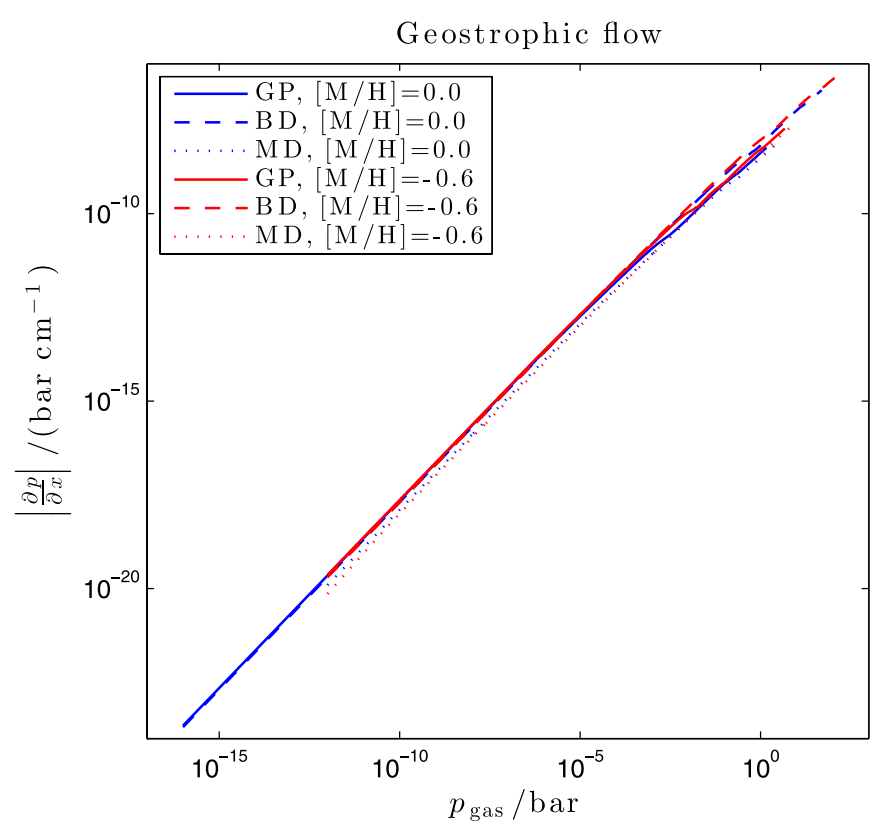

Figure 9. Pressure gradient criterion for a geostrophic flow (Equation (18)) for all three groups of low-mass objects considered here, with solar elemental abundances and low metallicity. This plot shows the minimum required pressure gradient needed to drive flows at the critical speed $\left(\left|u_{y}\right| \approx O\left(10 \mathrm{~km} \mathrm{~s}^{-1}\right)\right)$ needed for Alfvén ionization. We have assumed a typical low-mass object with rotation angular frequency $\omega=3.5 \times 10^{-4} \mathrm{rad} \mathrm{s}^{-1}(T=5 \mathrm{hr})$; the Coriolis parameter is $f \approx 4.9 \times 10^{-4} \mathrm{rad} \mathrm{s}^{-1}$ for $\phi=\pi / 4$ (mid-latitudes).

(A color version of this figure is available in the online journal.)

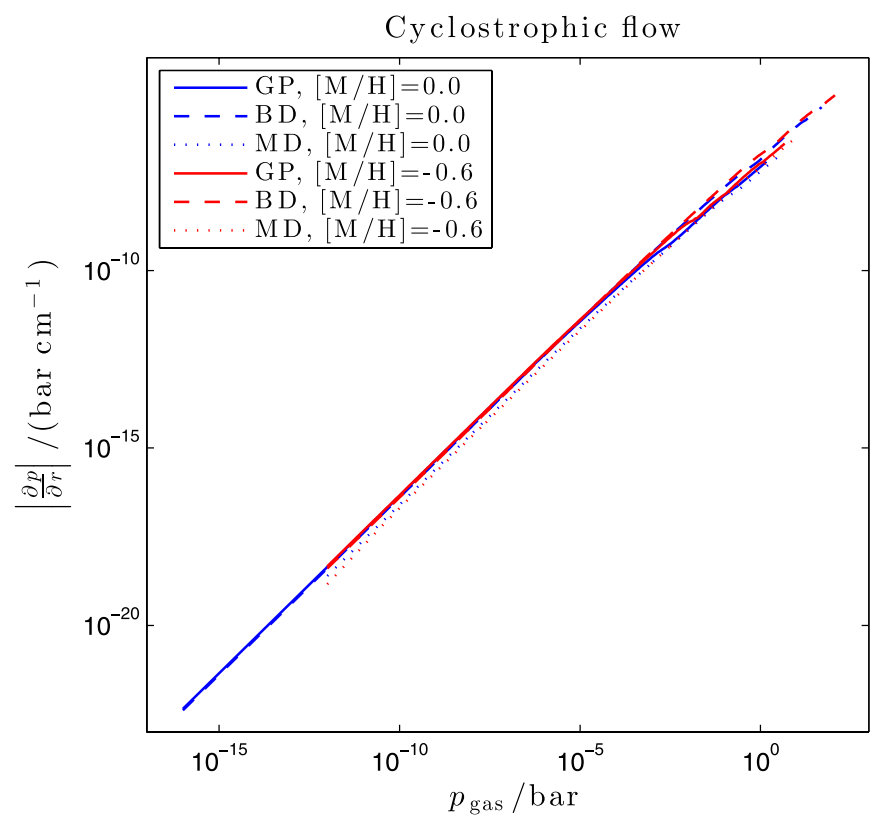

Figure 10. Pressure gradient criterion for a cyclostrophic flow (Equation (20)) for all three groups of low-mass objects considered here, with solar elemental abundances and low metallicity. This plot shows the minimum required pressure gradient needed to drive flows at the critical speed $\left(\left|u_{y}\right| \approx O\left(10 \mathrm{~km} \mathrm{~s}^{-1}\right)\right)$ needed for Alfvén ionization. We have assumed a typical low-mass object with rotation angular frequency $\omega=3.5 \times 10^{-4} \mathrm{rad} \mathrm{s}^{-1}(T=5 \mathrm{hr})$; the Coriolis parameter is $f \approx 4.9 \times 10^{-4} \mathrm{rad} \mathrm{s}^{-1}$ for $\phi=\pi / 4$ (mid-latitudes).

(A color version of this figure is available in the online journal.)

diffuse and the density is low $\rho_{\text {gas }} \approx 10^{-20}-10^{-17} \mathrm{~g} \mathrm{~cm}^{-3}$ the required pressure gradient to drive the needed flows is $\left|\partial p_{\text {gas }} / \partial x\right| \gtrsim\left(10^{-25}-10^{-18}\right)$ bar $\mathrm{cm}^{-1}$. At lower altitudes $\left(p_{\text {gas }} \approx 1-10^{2}\right.$ bar), the atmospheric density is clearly higher

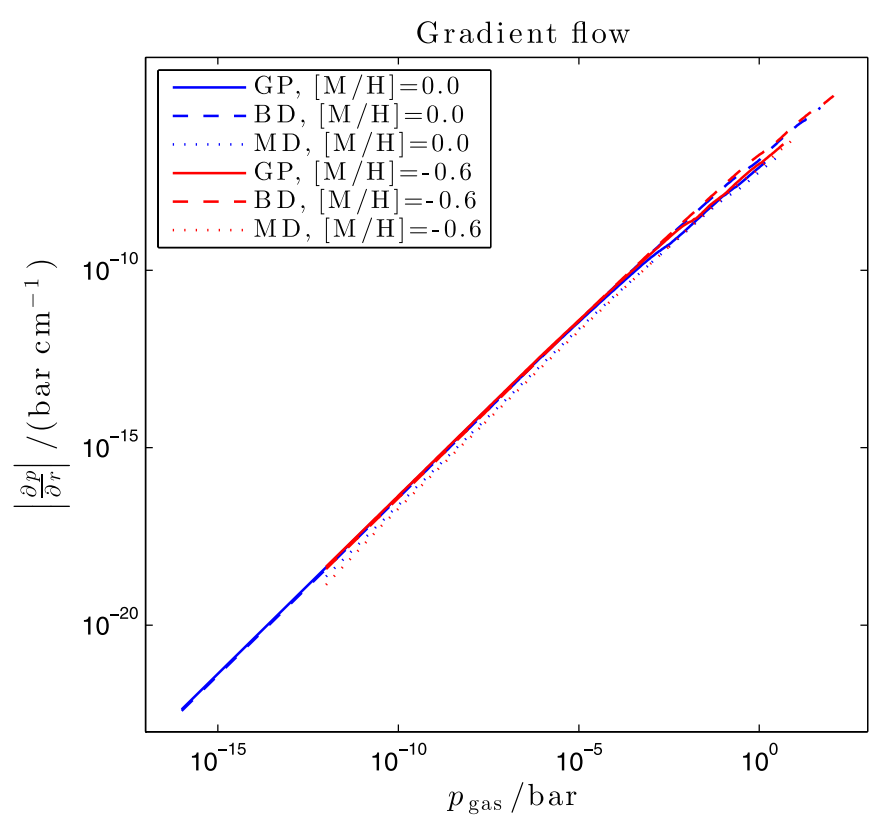

Figure 11. Pressure gradient criterion for a gradient flow (Equation (23)) for all three groups of low-mass objects considered here, with solar elemental abundances and low metallicity. This plot shows the minimum required pressure gradient needed to drive flows at the critical speed $\left(\left|u_{y}\right| \approx O\left(10 \mathrm{~km} \mathrm{~s}^{-1}\right)\right)$ needed for Alfvén ionization. We have assumed a typical low-mass object with rotation angular frequency $\omega=3.5 \times 10^{-4} \mathrm{rad} \mathrm{s}^{-1}(T=5 \mathrm{hr})$; the Coriolis parameter is $f \approx 4.9 \times 10^{-4} \mathrm{rad} \mathrm{s}^{-1}$ for $\phi=\pi / 4$ (mid-latitudes).

(A color version of this figure is available in the online journal.)

$\left(\rho_{\text {gas }} \approx 10^{-5}-10^{-3} \mathrm{~g} \mathrm{~cm}^{-3}\right.$ ) and so to obtain similar flows the required pressure gradient is much larger $\left(\left|\partial p_{\text {gas }} / \partial x\right| \approx\right.$ $\left.10^{-8}-10^{-6} \mathrm{bar} \mathrm{cm}^{-1}\right)$. Typically for the Earth, pressure gradients are of the order of $\left|\partial p_{\text {gas }} / \partial x\right| \approx 10^{-7}$ bar $\mathrm{cm}^{-1}$ in the troposphere or at mid-latitudes $\approx 10^{-9}$ bar $^{-1}$, with much greater pressure gradients expected at fronts. With this fact in mind, it seems plausible that in more exotic environments, namely, the atmosphere of a low-mass object, the pressure gradients, and hence wind speeds, required for Alfvén ionization are achievable.

Figures 9-11 show that at low atmospheric pressures the required pressure gradient criteria are smaller than at higher pressures; this geographic locale conveniently coincides with the region of the atmosphere where the criteria for a magnetized seed plasma are easiest to achieve (Figure 6).

Figures 9-11 show the dependence of the different flow regimes on the atmospheric density where a low-mass object rotating with a constant angular frequency $\omega=3.5 \times 10^{-4} \mathrm{rad} \mathrm{s}^{-1}$ ( $T=5 \mathrm{hr}$ and $f \approx 4.9 \times 10^{-4} \mathrm{rad} \mathrm{s}^{-1}$ for $\phi=\pi / 4$ ) is considered. However, geostrophic and gradient flow speeds also depend on the rotation period of the object (and hence the Coriolis parameter); Figure 12 is a plot of Equations (18) and (23) as a function of rotation period $T$ for a constant atmospheric density. Without loss of generality, we have chosen $\rho_{\text {gas }} \approx 10^{-10} \mathrm{~g} \mathrm{~cm}^{-3}$ since it is typical value for the density at mid-atmospheric pressures ( $p_{\text {gas }} \approx 10^{-5}$ bar) for the model atmospheres studied here (Figure 1). The pressure gradient criterion for geostrophic flows is linearly dependent on the Coriolis parameter $f=2 \omega \sin \phi$ and therefore inversely proportional to the period $T$ (Equation (18)). Thus, Figure 12 shows that for geostrophic flows, as the rotation period of the lowmass object increases the required pressure gradient for Alfvén ionization to occur decreases. Therefore, it is relatively easier 


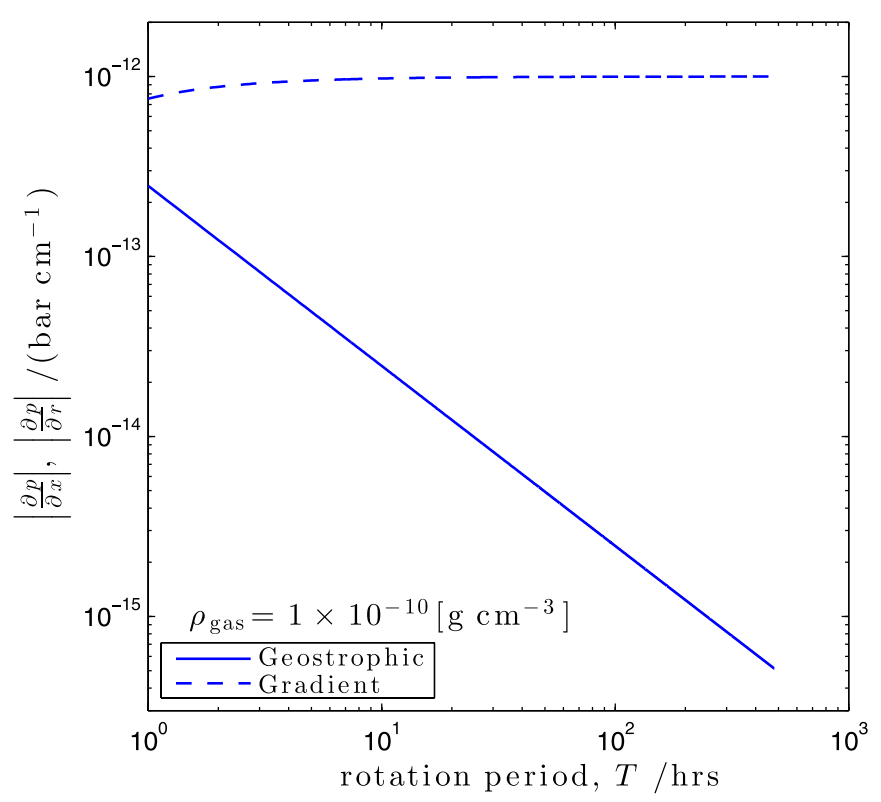

Figure 12. Pressure gradient as a function of rotational period $T$ for a geostrophic flow (Equation (18); solid line) and a gradient flow (Equation (23); dashed line). This gradient is the critical pressure gradient required to provide large enough local gas flow speeds to allow Aflvén ionization.

(A color version of this figure is available in the online journal.)

for slower rotating objects to meet the flow speed criterion. For example, for an object with a rotation period $T \approx 2 \mathrm{hr}$, the pressure gradient must exceed $\approx 10^{-9}$ bar $\mathrm{cm}^{-1}$; whereas for an object with $T \approx 10^{2} \mathrm{hr},\left|\partial p_{\text {gas }} / \partial x\right| \gtrsim 10^{-11} \mathrm{bar} \mathrm{cm}^{-1}$. For gradient flows, as $T$ increases so does the required minimum pressure gradient until it saturates at a maximum value $\left(\left|\partial p_{\text {gas }} / \partial r\right| \gtrsim 10^{-12}\right.$ bar $\left.\mathrm{cm}^{-1}\right)$. In the limit of large $T$ (i.e., $f \rightarrow 0$ ), Equation (23) becomes $\left|\partial p_{\text {gas }} / \partial r\right| \gtrsim 10^{6} \rho_{\text {gas }} / r$; there-

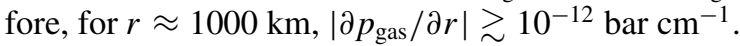

\section{DEGREE OF IONIZATION FROM ALFVÉN IONIZATION}

For Alfvén ionization, we require that (1) the seed plasma is strongly magnetized and (2) the neutral gas flow reaches a critical speed $v_{c}$. Assuming the required conditions can be met and that the neutral gas flow speed can be maintained, Alfvén ionization can ionize the entirety of the gas in a localized volume, leaving a plasma with an electron number density equal to the gas component number density (assuming $100 \%$ ionization) plus the initial seed magnetized plasma number density. This statement assumes that the gas is singly ionized. $100 \%$ ionization via the Alfvén mechanism is perfectly feasible and has been demonstrated for hydrogen in laboratory experiments (Fahleson 1961). Here, we are interested in Alfvén ionization in the atmospheres of gas giant planets, brown dwarfs, and M-dwarfs. Therefore, we assume that the seed plasma is sourced from the ions and electrons created by thermal ionization in the atmosphere and we exclude any contributions from additional sources such as inter-grain discharges and cosmic rays. In a gas mixture composed of a number of species, the electron number density cannot exceed the target species neutral number density plus the initial seed electron number density. For a given target species, this requirement can enhance the local degree of ionization $f_{e}=n_{i} /\left(n_{i}+n_{\mathrm{gas}}\right)$ beyond $10^{-7}$, resulting in an ionized gas that can be treated as a plasma.
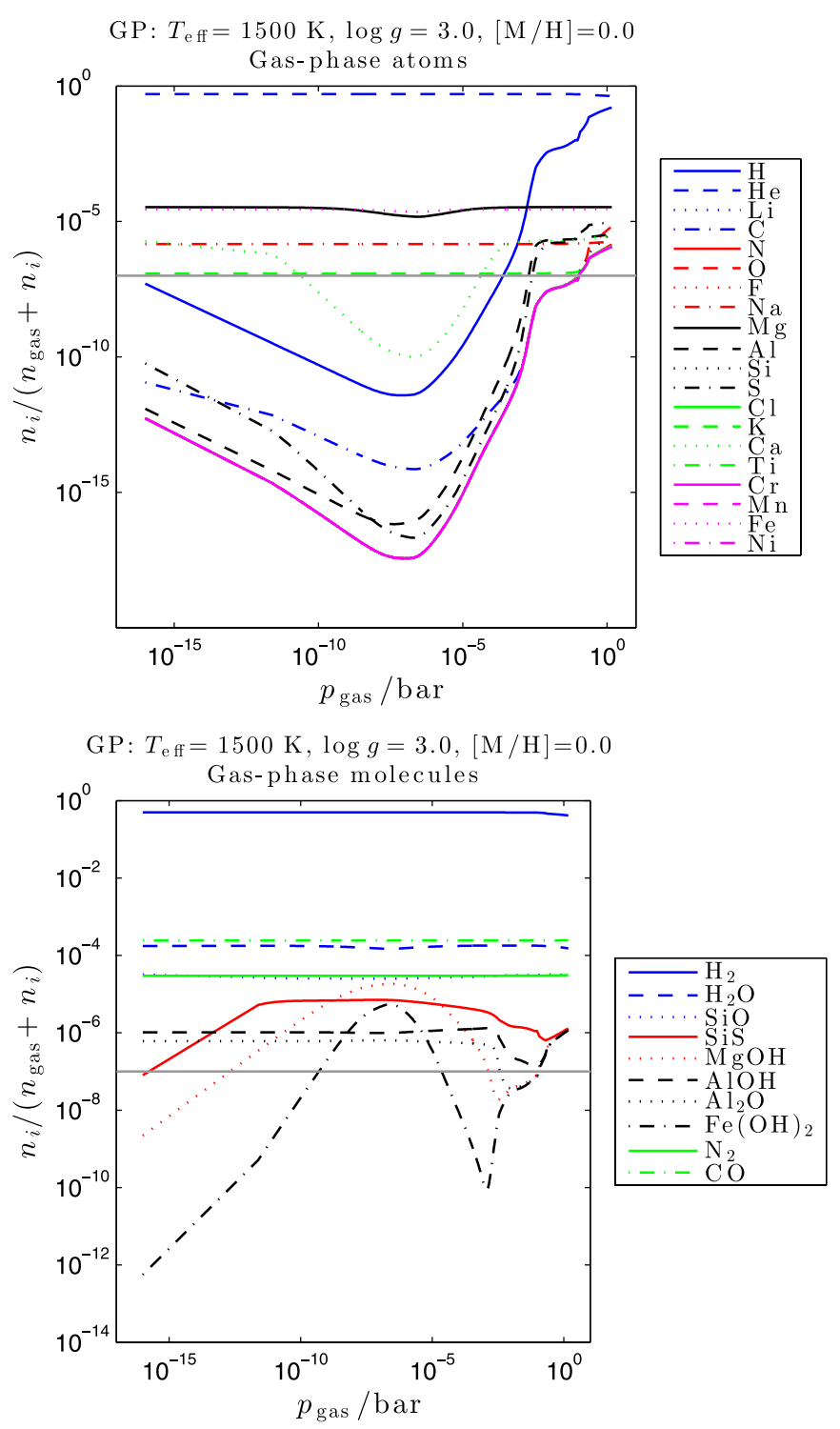

Figure 13. Degree of ionization $f_{e}=n_{i} /\left(n_{\text {gas }}+n_{i}\right)$ resulting from Alfvén ionization for a gas giant planet (GP: $T_{\mathrm{eff}}=1500 \mathrm{~K}, \log g=3.0$ ) for individual species being entirely ionized in a localized atmospheric pocket, assuming initially solar elemental abundances $([\mathrm{M} / \mathrm{H}]=0.0)$. The top plot shows $f_{e}$ for atoms; the bottom plot shows $f_{e}$ for selected molecules. The gray line indicates $f_{e}=10^{-7}$, the degree of ionization required to constitute a plasma.

(A color version of this figure is available in the online journal.)

Figures 13-18 show the resulting degrees of ionization from Alfvén ionization, if specific individual species constituting the atmospheric gas are entirely ionized (on their own) in a localized atmospheric pocket, assuming solar elemental abundances $[\mathrm{M} / \mathrm{H}]=0.0$ (Figures 13-15) and a lower metallicity of $[\mathrm{M} / \mathrm{H}]=-0.6$ (Figures 16-18). In general, if in a localized atmospheric pocket a particular species can be $100 \%$ ionized, then the species with the greatest number density will yield the highest degree of ionization. To summarize, if entirely ionized on their own, $\mathrm{He}, \mathrm{Fe}, \mathrm{Mg}, \mathrm{Na}, \mathrm{H}_{2}, \mathrm{CO}, \mathrm{H}_{2} \mathrm{O}, \mathrm{N}_{2}$, and $\mathrm{SiO}$ all consistently increase the degree of ionization beyond $10^{-7}$ throughout the model atmospheres considered here.

In all the atmosphere models studied here, $\mathrm{He}, \mathrm{Fe}, \mathrm{Mg}$, and $\mathrm{Na}$, if individually ionized $(100 \%)$ in a localized atmospheric volume, increase the degree of ionization sufficiently high $\left(f_{e} \approx 1,10^{-5}, 10^{-5}\right.$, and $10^{-6}$, respectively) such that the 

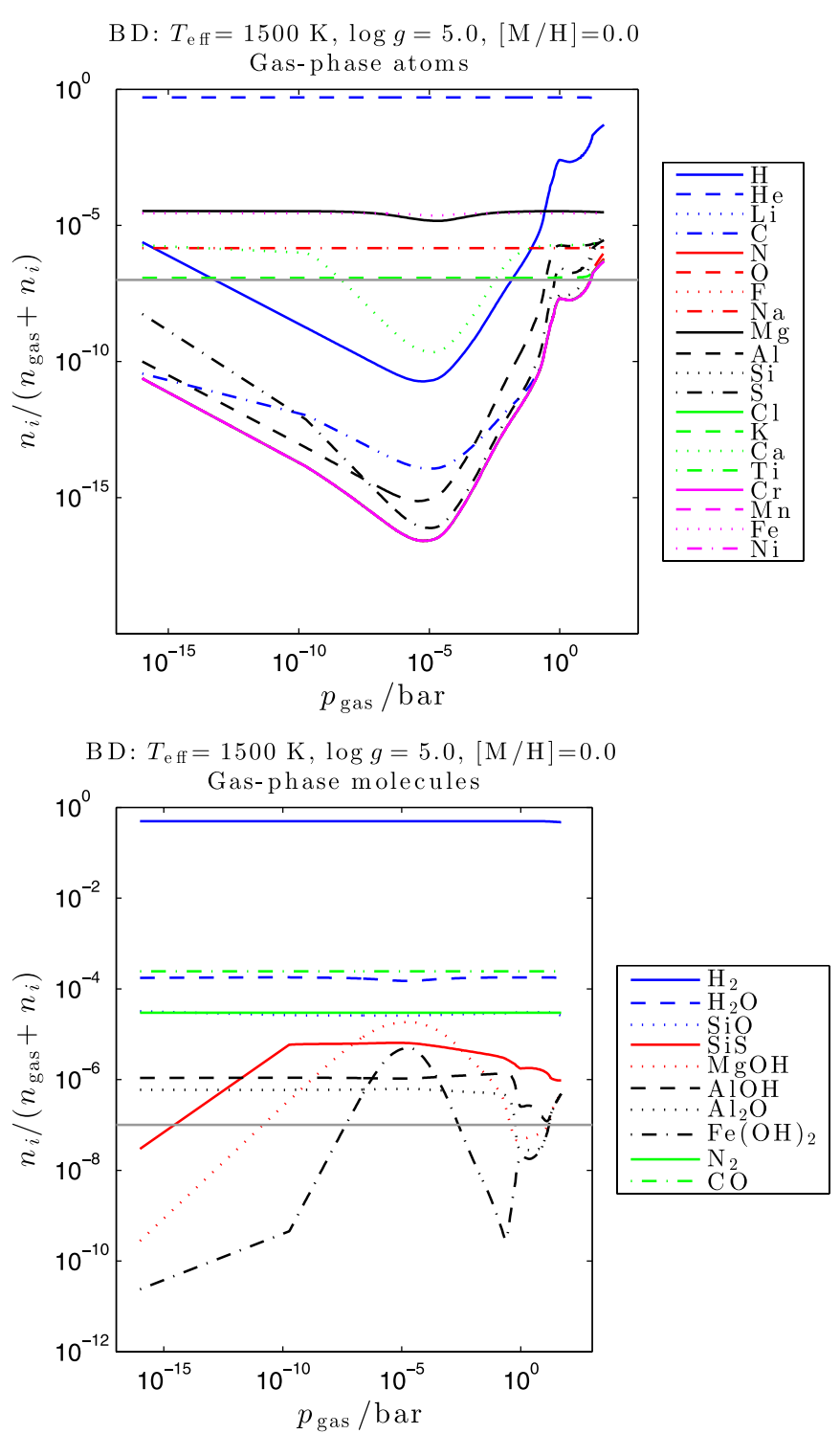

Figure 14. Degree of ionization $f_{e}=n_{i} /\left(n_{\mathrm{gas}}+n_{i}\right)$ resulting from Alfvén ionization for a brown dwarf (BD: $T_{\text {eff }}=1500 \mathrm{~K}, \log g=5.0$ ) for individual species being entirely ionized in a localized atmospheric pocket, assuming initially solar elemental abundances $([\mathrm{M} / \mathrm{H}]=0.0)$. The top plot shows $f_{e}$ for atoms; the bottom plot shows $f_{e}$ for selected molecules. The gray line indicates $f_{e}=10^{-7}$, the degree of ionization required to constitute a plasma.

(A color version of this figure is available in the online journal.)

ionized gas can be considered a plasma $\left(f_{e} \geqslant 10^{-7}\right)$ throughout the atmosphere. The critical ionization speed that a streaming neutral gas must obtain for the ionization of these respective atoms is $v_{c}=34.43 \mathrm{~km} \mathrm{~s}^{-1}, 5.22 \mathrm{~km} \mathrm{~s}^{-1}, 7.79 \mathrm{~km} \mathrm{~s}^{-1}$, and $6.57 \mathrm{~km} \mathrm{~s}^{-1}$. $\mathrm{He}, \mathrm{Fe}, \mathrm{Mg}$, and $\mathrm{Na}$ have the greatest number density of the atomic chemical elements in the atmospheres. In addition, $\mathrm{Mg}$ and $\mathrm{Fe}$ play a key role in the formation of dust clouds, which is evident from a dip in their atmospheric $\left(p_{\text {gas }}, T_{\text {gas }}\right)$ profile in the cloud forming region (Figure 1). Helium is inert and so is not affected by the formation of clouds and its number density does not vary significantly from the initial, prescribed abundance. However, although chemically inert, helium metastable states can affect the energy budget of the system under consideration by momentarily locking energy in an excited state. Note that the number density of atomic $\mathrm{He}$ is greater than that of $\mathrm{H}$; molecular hydrogen $\mathrm{H}_{2}$ is the dominant
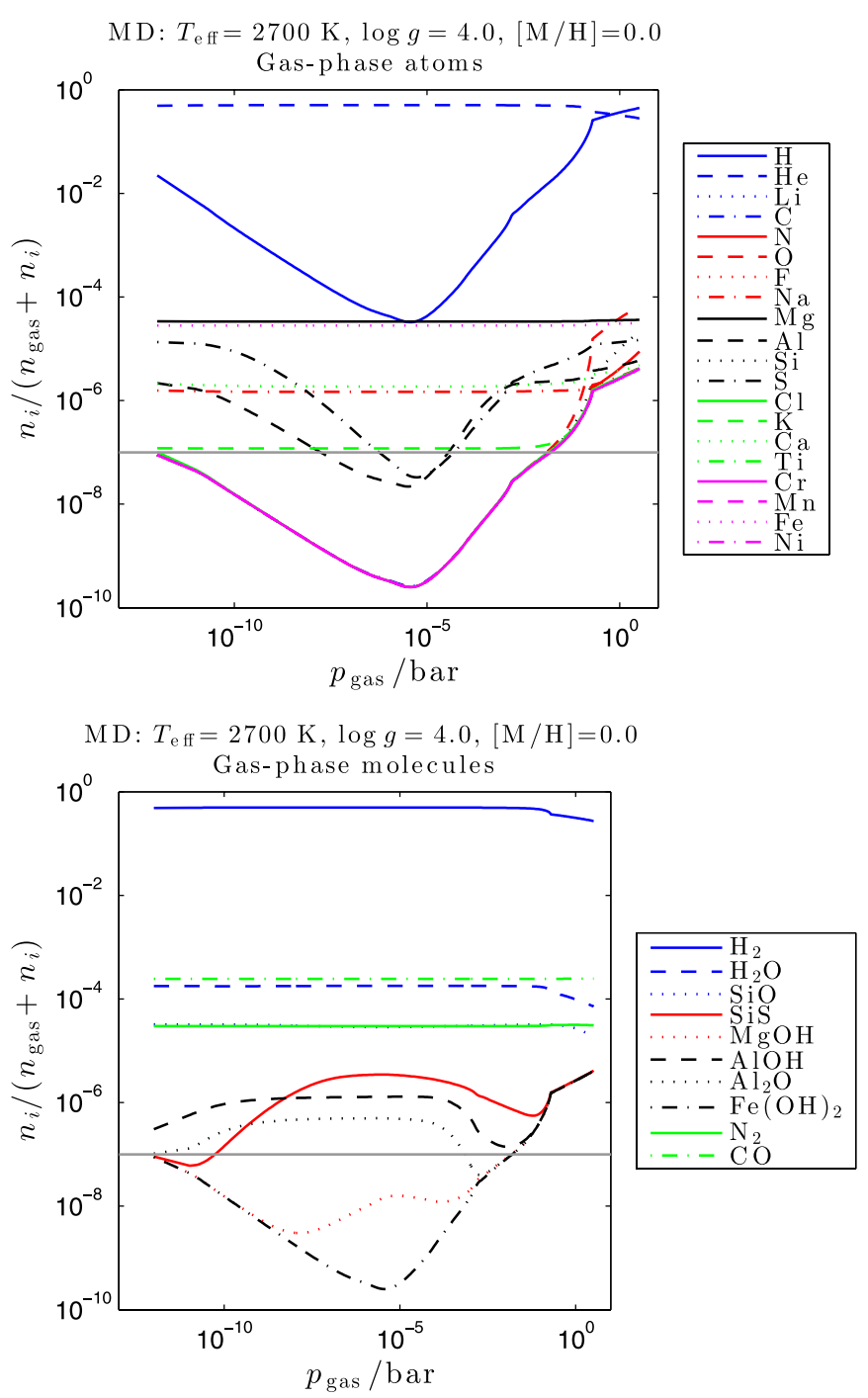

Figure 15. Degree of ionization $f_{e}=n_{i} /\left(n_{\mathrm{gas}}+n_{i}\right)$ resulting from Alfvén ionization for a M-dwarf (MD: $T_{\mathrm{eff}}=2700 \mathrm{~K}, \log g=4.0$ ) for individual species being entirely ionized in a localized atmospheric pocket, assuming initially solar elemental abundances $([\mathrm{M} / \mathrm{H}]=0.0)$. The top plot shows $f_{e}$ for atoms; the bottom plot shows $f_{e}$ for selected molecules. The gray line indicates $f_{e}=10^{-7}$, the degree of ionization required to constitute a plasma.

(A color version of this figure is available in the online journal.)

$\mathrm{H}$-carrier, the formation of which depletes the atomic $\mathrm{H}$ in all the atmospheres considered here for $p_{\text {gas }} \lesssim 10^{-1}$ bar.

The low-metallicity model atmospheres $([\mathrm{M} / \mathrm{H}]=-0.6$; Figures 16-18) have a lower number density of metals relative to hydrogen and so the degree of ionization from these metals is lower than in the solar metallicity case since their number density is reduced. Potassium $\left(v_{c}=4.22 \mathrm{~km} \mathrm{~s}^{-1}\right)$ can also be significant in enhancing the degree of ionization to form an atmospheric plasma, but only in solar abundance models (Figures 13-15); in the low-metallicity models, the degree of ionization falls below the threshold value of $10^{-7}$ over the majority of the atmosphere, only exceeding $10^{-7}$ at very high pressures (in all three groups of low-mass object considered here) and very low pressures (MD only; Figures 15 and 18). Calcium $\left(v_{c}=5.42 \mathrm{~km} \mathrm{~s}^{-1}\right)$ amplifies the degree of ionization beyond $10^{-7}$ for all pressures in MD atmospheres (even for a low metallicity atmosphere), but only for high and low pressures in GP atmospheres (Figures 13 and 16) and BD atmospheres (Figures 14 and 17), which are considerably cooler than the 

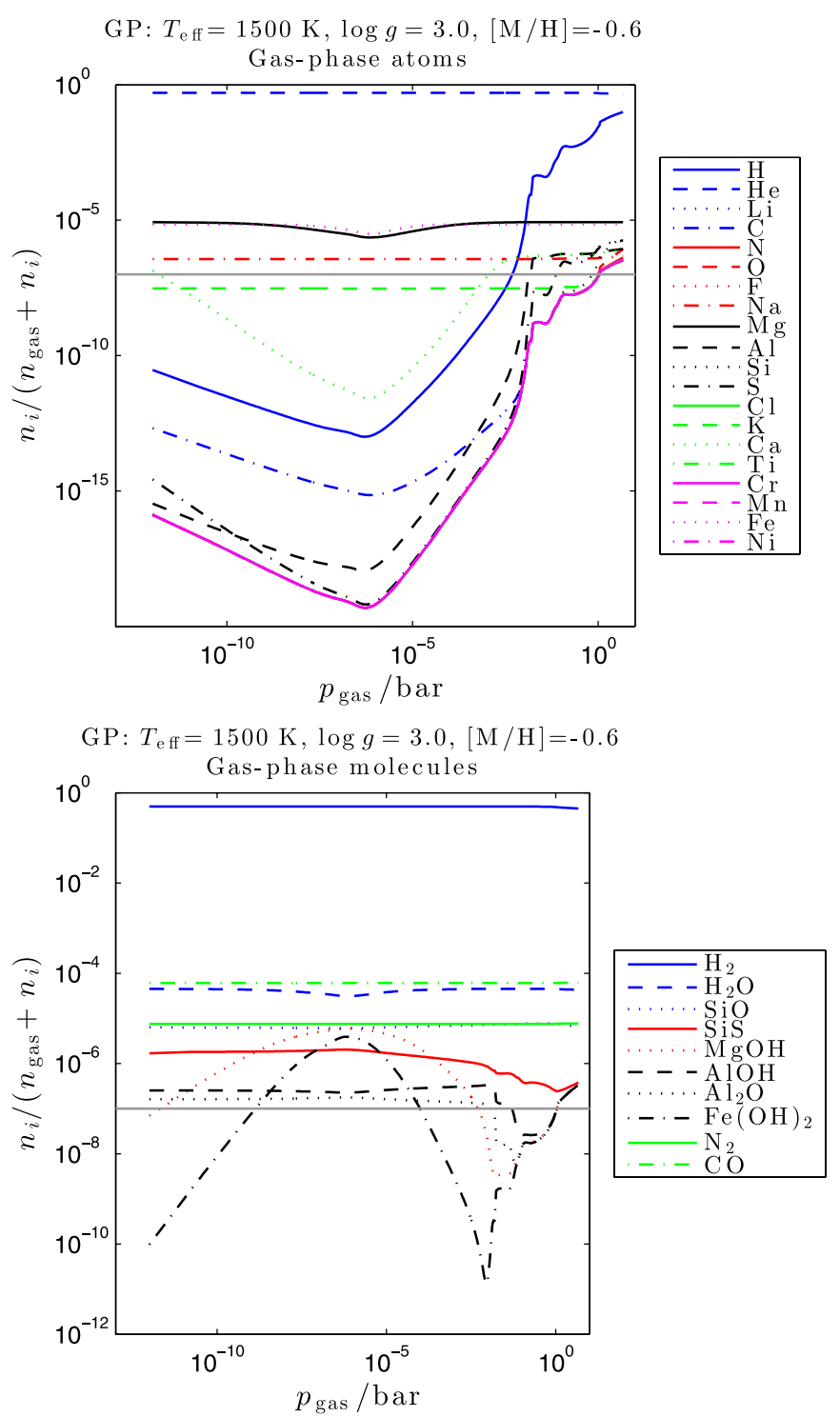

Figure 16. Degree of ionization $f_{e}=n_{i} /\left(n_{\text {gas }}+n_{i}\right)$ resulting from Alfvén ionization for a gas giant planet (GP: $T_{\text {eff }}=1500 \mathrm{~K}, \log g=3.0$ ) for individual species being entirely ionized in a localized atmospheric pocket, assuming an initially low metallicity $([\mathrm{M} / \mathrm{H}]=-0.6)$. The top plot shows $f_{e}$ for atoms; the bottom plot shows $f_{e}$ for selected molecules. The gray line indicates $f_{e}=10^{-7}$, the degree of ionization required to constitute a plasma.

(A color version of this figure is available in the online journal.)

M-dwarf model atmosphere. For the remaining elements, their relative number density in the gas phase is so small that if they are entirely ionized, they, on their own, only contribute significantly to the generation of a plasma at high atmospheric pressures.

Whereas the GP (Figures 13 and 16) and BD (Figures 14 and 17) models show a similar $f_{e}$ behavior, the MD model (Figures 15 and 18) shows significant differences. For example, after helium, hydrogen yields the highest degree of ionization $\left(f_{e} \approx 10^{-4}\right.$ at $\left.p_{\text {gas }} \approx 10^{-5}\right)$, which is not the case in the GP and $\mathrm{BD}$ atmospheric models. Moreover, in contrast to the GP and BD models, calcium, aluminum, and sulfur yield a degree of ionization greater than $10^{-7}$ for most atmospheric pressures. In low metallicity MD atmospheres $([\mathrm{M} / \mathrm{H}]=-0.6)$, additional elements such as $\mathrm{O}$ and $\mathrm{Cl}$ (as well as other trace elements) become significant in their contribution to $f_{e}$ at low pressures $\left(p_{\text {gas }} \lesssim 10^{-10}\right.$ bar).
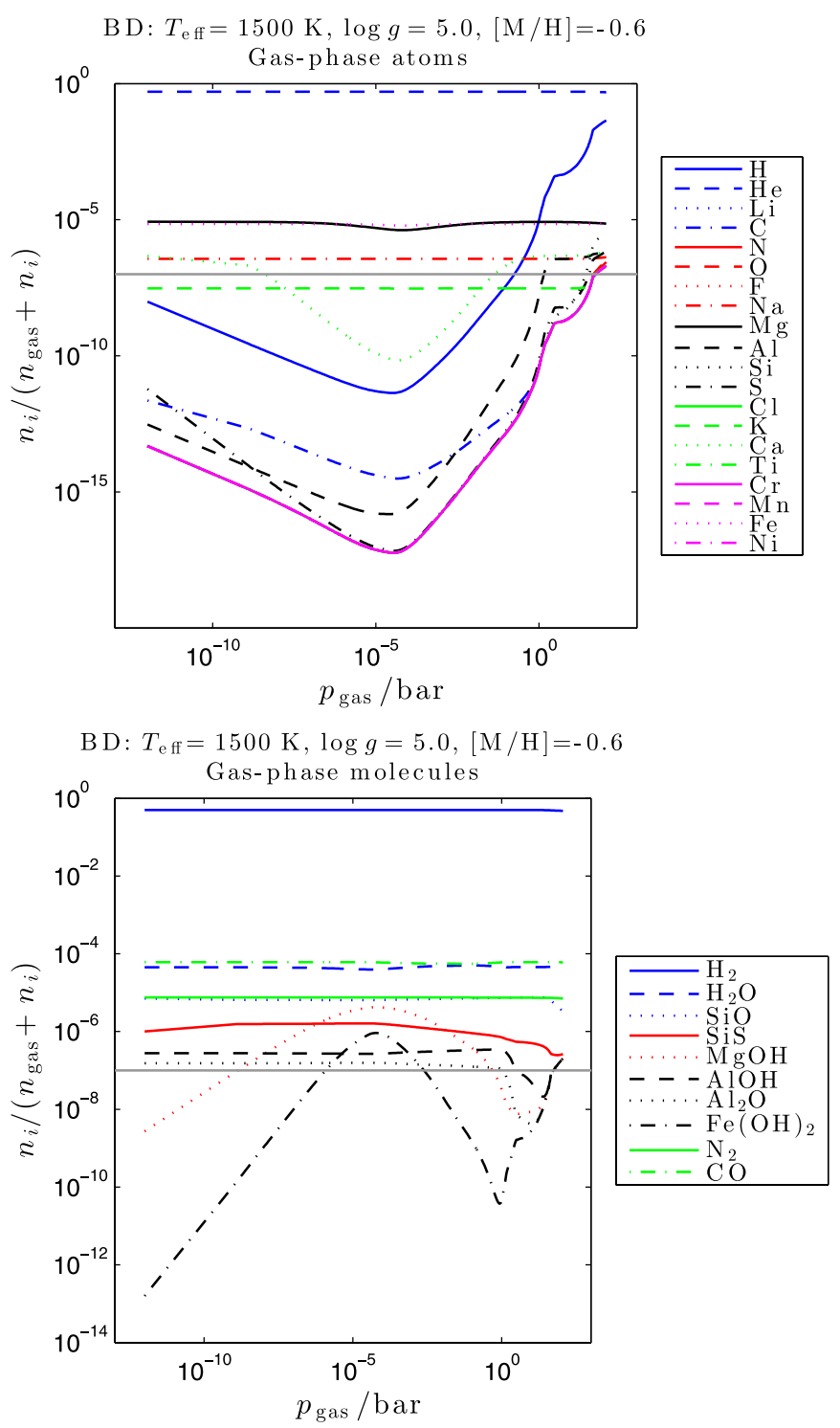

Figure 17. Degree of ionization $f_{e}=n_{i} /\left(n_{\mathrm{gas}}+n_{i}\right)$ resulting from Alfvén ionization for a brown dwarf (BD: $T_{\text {eff }}=1500 \mathrm{~K}, \log g=5.0$ ) for individual species being entirely ionized in a localized atmospheric pocket, assuming an initially low metallicity $([\mathrm{M} / \mathrm{H}]=-0.6)$. The top plot shows $f_{e}$ for atoms; the bottom plot shows $f_{e}$ for selected molecules. The gray line indicates $f_{e}=10^{-7}$, the degree of ionization required to constitute a plasma.

(A color version of this figure is available in the online journal.)

For molecular species, the situation is more complicated (see the lower panels of Figures 13-18). In our analysis of oxygenrich, ultra-cool atmospheres of low-mass objects, we have focused on several key molecules, e.g., $\mathrm{H}_{2}$ for its high number density, $\mathrm{H}_{2} \mathrm{O}$ for its spectral importance, and $\mathrm{CO}$ because it is minimally affected by the formation of dust clouds. In general, as in the atomic case, the low-metallicity models (Figures 16-18) yield lower degrees of ionization for the participating molecules in comparison with the solar abundance cases (Figures 13-15). In all the atmospheric models considered, $\mathrm{H}_{2}, \mathrm{CO}, \mathrm{H}_{2} \mathrm{O}, \mathrm{N}_{2}$, and $\mathrm{SiO}$ all yield $f_{e} \geqslant 10^{-7}$ for all atmospheric pressures $\left(f_{e} \approx 1,10^{-4}, 10^{-4}, 10^{-5}\right.$, and $10^{-5}$, respectively) and so these species would successfully form a molecular plasma. $\mathrm{AlOH}$ and $\mathrm{Al}_{2} \mathrm{O}$ consistently yield $f_{e} \approx 10^{-6}$, except at high pressures in low metal GP and BD models (Figures 16 and 17) and in MD atmospheres (Figures 15 and 18). At high atmospheric pressures, the local temperature is high enough to dissociate $\mathrm{AlOH}$ and 

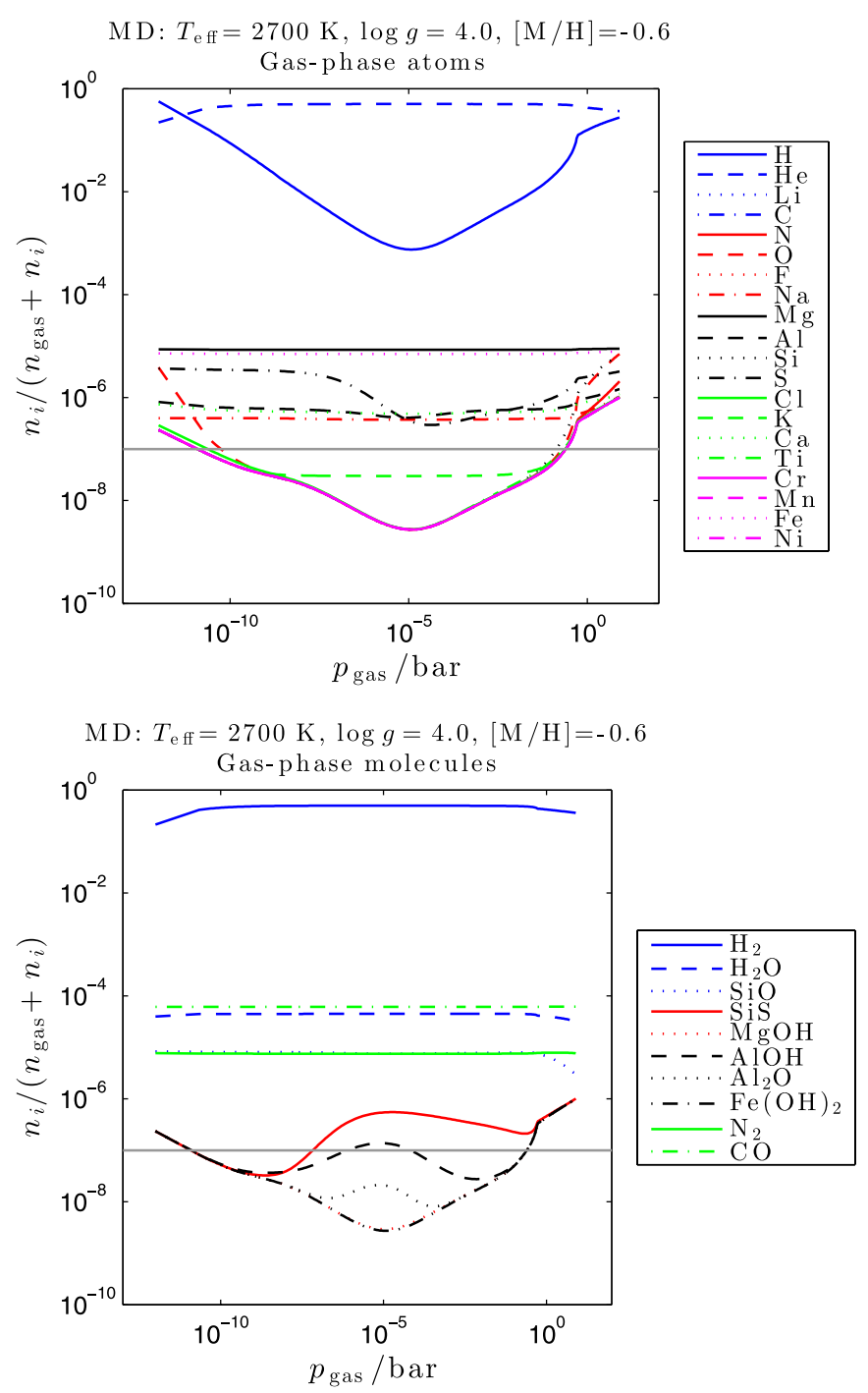

Figure 18. Degree of ionization $f_{e}=n_{i} /\left(n_{\text {gas }}+n_{i}\right)$ resulting from Alfvén ionization for an M-dwarf (MD: $T_{\text {eff }}=2700 \mathrm{~K}, \log g=4.0$ ) for individual species being entirely ionized in a localized atmospheric pocket, assuming an initially low metallicity $([\mathrm{M} / \mathrm{H}]=-0.6)$. The top plot shows $f_{e}$ for atoms; the bottom plot shows $f_{e}$ for selected molecules. The gray line indicates $f_{e}=10^{-7}$, the degree of ionization required to constitute a plasma.

(A color version of this figure is available in the online journal.)

$\mathrm{Al}_{2} \mathrm{O}$ molecules, reducing their number density and hence the degree of ionization resulting from Alfvén ionization. Similarly, $\mathrm{SiS}$ produces modest degrees of ionization $f_{e} \approx 10^{-6}$, except at low atmospheric pressures in low-metallicity MD model atmospheres (Figure 18), where $f_{e}<10^{-7}$.

\section{DISCUSSION}

Observations of radio and X-ray emission suggest that such objects harbor an atmospheric, magnetized plasma. This result further suggests that substellar atmospheres are composed of gas-plasma mixtures that participate in a range of plasma phenomena such as waves, flows, and instabilities. In a plasma, dust becomes charged and can grow (forming clouds) electrostatically via ion deposition. These charged dust particles will be susceptible to inter-grain electrical discharges that will energize the ambient gas-plasma and will participate in other collective plasma processes. Therefore, it is key to our understanding of substellar atmospheres that the relevant plasma phenomena are incorporated into atmospheric models and that their observational consequences are quantified. Moreover, it is critical to identify the ionization processes that create and replenish the atmospheric plasma.

The primary aim of this paper was to show that Alfvén ionization is a significant process that can occur in the atmospheres of low-mass objects, responsible for the production of ionized pockets of gas that have a degree of ionization $\geqslant 10^{-7}$, qualifying them as atmospheric plasmas. A plasma is defined as a collection of charged particles of sufficiently high number density that the Coulomb force is significant in determining its properties, yet sufficiently dilute such that the nearest neighbor interaction is dominated not by binary collisions, but instead by the collective electromagnetic influence of the many distant particles. For an ionized gas to behave like a plasma rather than a neutral gas, the degree of ionization should be $\geqslant 10^{-7}$. The presence of pockets of atmospheric magnetized plasma opens the door to a multitude of plasma processes not yet considered in current atmospheric models, such as the growth of dust grains via ion deposition (Stark et al. 2006).

This paper has outlined the criteria required for Alfvén ionization to occur and has demonstrated its application using example atmospheres of low-mass objects such as giant gas planets $\left(\log g=3.0, T_{\text {eff }}=1500 \mathrm{~K}\right)$, brown dwarfs $\left(\log g=5.0, T_{\text {eff }}=1500 \mathrm{~K}\right)$, and M-dwarfs $(\log g=4.0$, $\left.T_{\text {eff }}=2700 \mathrm{~K}\right)$, for both solar $([\mathrm{M} / \mathrm{H}]=0.0)$ and sub-solar $([\mathrm{M} / \mathrm{H}]=-0.6)$ metallicities.

Alfvén ionization requires (1) a low-density, magnetized seed plasma initially present in the atmosphere and (2) a neutral gas flow that attains a critical speed $v_{c}$. Processes naturally occurring in the atmosphere such as thermal ionization, local discharge events (lightning), and cosmic-ray ionization easily provide enough electrons and ions to constitute a seed plasma. For the seed plasma to be magnetized, an ambient magnetic field must be present and be sufficiently strong such that the plasma dynamics are influenced by it. For the model atmospheres considered here at high altitudes $\left(p_{\text {gas }} \approx 10^{-12}\right.$ bar), $B \gtrsim 10^{-6} \mathrm{G}$ is required locally, whereas at lower altitudes the criterion is much more severe $B \gtrsim 10^{6} \mathrm{G}$; however, over a large atmosphere pressure range $\left(p_{\text {gas }} \lesssim 10^{-2}\right.$ bar), the magnetized plasma criterion is easily achievable and the minimum magnetic flux density required locally is $\lesssim 10^{4} \mathrm{G}$. Typical average, global magnetic flux densities of gas planets, brown dwarfs, and M-dwarfs ( $\approx 10 \mathrm{G}$ for GPs and $1 \mathrm{kG}$ for BDs and MDs) imply that the seed plasma is adequately magnetized and that only at high pressures is a significant magnetic flux density required. Due to the weaker magnetic field strength of GPs, it is harder to ensure that the seed plasma is magnetized. Therefore, Alfvén ionization is easier to achieve in BDs and MDs. However, other processes can occur in the exoplanetary environment that can enhance the ambient magnetic field (e.g., Khodachenko et al. 2012 discuss the formation of magnetodisks), aiding Alfvén ionization.

For Alfvén ionization, the neutral gas flow speed must reach the critical flow speed $v_{c}$, which, for most gas species of interest, $v_{c} \approx O\left(1-10 \mathrm{~km} \mathrm{~s}^{-1}\right)$. The calculations presented here considered three standard meteorological balanced flows (geostrophic, cyclostrophic, and gradient) and found that reasonably low pressure gradients ranging from $\approx 10^{-18}-10^{-7} \mathrm{bar}^{-1}$ are sufficient to drive the required neutral gas flows for Alfvén ionization. For the Earth, typical pressure gradients are of the order $\left|\partial p_{\text {gas }} / \partial x\right| \approx 10^{-7}$ bar $\mathrm{cm}^{-1}$ in the troposphere or $\approx 10^{-9}$ bar $\mathrm{cm}^{-1}$ at mid-latitudes, with much greater 
pressure gradients expected at fronts. Bearing this fact in mind, it seems plausible that in more exotic environments such as the atmosphere of a low-mass object, such pressure gradients and associated wind speeds required for Alfvén ionization are achievable. Our analysis shows that at low atmospheric pressures the balanced flow criteria are smaller than at higher pressures, coinciding with the region of the atmosphere where the criteria for a magnetized seed plasma are most favorable. In contrast with the other atmospheric models, GP and BD models for $[\mathrm{M} / \mathrm{H}]=0.0$ extend to lower mass densities and pressures and so it is easier to fulfill the criteria for the balanced flows discussed here in these atmospheres where the pressure is low.

If the criteria for Alfvén ionization can be met, then the process can ionize the entirety of the gas in a localized atmospheric volume, creating a plasma with an electron number density equal to the target neutral species number density plus the initial seed plasma number density. In general, if in a localized atmospheric pocket a particular species can be $100 \%$ ionized, then the species with the greatest number density will yield the highest degree of ionization. 100\% ionization via the Alfvén mechanism is perfectly feasible and has been demonstrated for hydrogen in laboratory experiments (Fahleson 1961). Alfvén ionization operates best at mid to high altitudes (mid to low atmospheric pressures), where the seed plasma is easier to magnetize and the pressure gradients required to drive the required neutral flows are the smallest. For the model atmospheres considered here, our results show that $\mathrm{He}, \mathrm{Fe}$, $\mathrm{Mg}$, and $\mathrm{Na}$, if ionized, increase the degree of ionization of the local environment beyond $10^{-7}\left(f_{e} \approx 1,10^{-5}, 10^{-5}\right.$, and $10^{-6}$, respectively) such that the ionized gas will be a plasma. Furthermore, our calculations infer that it is easier to create a plasma with a higher degree of ionization in atmospheres with solar metallicities than with sub-solar metallicities.

Molecular plasmas are also expected to form in substellar atmospheres and can be easier to ionize in comparison to atoms since the required $v_{c}$ tends to be lower due to their larger mass and comparable ionization potential. For the selected molecules studied here, we find that Alfvén ionization of $\mathrm{H}_{2}$, $\mathrm{CO}, \mathrm{H}_{2} \mathrm{O}, \mathrm{N}_{2}$, and $\mathrm{SiO}$ all yield $f_{e} \geqslant 10^{-7}$ for the model atmospheres considered. However, in some cases, the ionization energy may exceed the dissociation energy of the molecule, increasing the likelihood that through the Alfvén ionization mechanism certain molecules will be destroyed, lowering their local number density. For example, $\mathrm{CO}$ has a dissociation energy of $11.13 \mathrm{eV}$ (at $0 \mathrm{~K}$ ) and an ionization potential of $14.0 \mathrm{eV}$, implying that there is a chance that $\mathrm{CO}$ will be dissociated before it can be ionized. For more complex molecules, total dissociation into neutral atoms will most likely occur in multiple stages, e.g., $\mathrm{H}_{2} \mathrm{O} \rightarrow \mathrm{OH}+\mathrm{H}$ and then $\mathrm{OH} \rightarrow \mathrm{O}+\mathrm{H}$, with the energy required for each stage being $5.08 \mathrm{eV}$ and $4.41 \mathrm{eV}$ (at $0 \mathrm{~K}$ ), respectively. Furthermore, additional processes affecting the atmospheric chemistry can occur such as molecular dissociative recombination or dissociative attachment.

The degree of ionization as a result of the Alfvén ionization process easily outstrips that from thermal ionization, where $f_{\mathrm{e} \text {,therm }} \leqslant 10^{-8}$ for most atmospheric pressures. At high atmospheric pressures, where the temperature increases, $f_{\mathrm{e}, \text { therm }}$ will be enhanced, but never to levels equivalent to those obtained from Alfvén ionization. Cosmic-ray ionization can yield higher degrees of ionization $\left(f_{\mathrm{e}, \text { cosmic }} \approx 10^{-8}\right)$ relative to thermal considerations, but still cannot compete with the degree of ionization from Alfvén ionization. In addition to Alfvén ionization, inter-grain electrical discharges will enhance the electron number density, boosting the local degree of ionization (Helling et al. 2011b).

Alfvén ionization also has important consequences for the subsequent chemistry that occurs in the atmospheres of lowmass objects due to the injection of a significant amount of electrons, positive ions, and radicals. This fact allows access to more complex chemistry, such as electron-moderated chemistry, that is otherwise not permitted if only thermal processes are available. The presence of an amplified free electron population increases the likelihood that these electrons attach themselves to the ambient neutral atoms, molecules, or dust particles, thereby enhancing the consequent chemistry, including the formation of dust or the formation of large-scale charge separations that may lead to lightning (Helling et al. 2013).

Charged particle surfaces grow via the accumulation of neutral atoms and an electrostatically attracted ion flux (and also polarizable molecules, which will be attracted by the unbalanced charge as well) and so will grow faster than the uncharged scenario when there is a neutral flux only. This statement assumes that the neutral flux remains the same and is not reduced by the creation of ions (at the expense of the neutrals) more than the charged grains' enhanced ion flux. The excitation of metastable states can also affect the chemistry by momentarily locking energy in an excited state and therefore impacting the energy budget of the system under consideration. An observable consequence of a metastable population will be the presence of forbidden lines in observed spectra, which would otherwise be absent in the non-ionizing case. For example, the metastable state $2^{3} S_{1}\left(E=19.82 \mathrm{eV}, v \approx 10^{15} \mathrm{~Hz}\right)$ of He may produce a forbidden line in the spectra.

If low-mass object atmospheres harbor a magnetized plasma, then it could be observable. The motion and mutual interaction of the plasma particles would manifest itself as continuum bremsstrahlung emission with a characteristic spectrum depending on the nature of the source. For example, the power radiated per unit volume per unit solid angle per unit angular frequency interval (the bremsstrahlung emission coefficient, $\epsilon_{\omega}$ ) for a thermal plasma is given by

$$
\begin{aligned}
\epsilon_{\omega}\left(T_{e}\right)= & \frac{8}{3 \sqrt{3}} \frac{Z^{2} n_{e} n_{i}}{m_{e}^{2} c^{3}}\left(\frac{e^{2}}{4 \pi \epsilon}\right)^{3}\left(\frac{m_{e}}{2 \pi k_{B} T_{e}}\right)^{1 / 2} \bar{g}\left(\omega, T_{e}\right) \\
& \times \exp \left(-\frac{\hbar \omega}{k_{B} T_{e}}\right)
\end{aligned}
$$

where $\bar{g}$ is the Gaunt factor, $Z_{i}$ is the ion charge number, $\omega$ is the photon angular frequency, and $n_{e}$ and $n_{i}$ are the electron and ion number density of the plasma, respectively (Boyd \& Sanderson 2003). In the regime $\hbar \omega \geqslant k_{B} T_{e}$, the slope of a loglinear plot of the bremsstrahlung emission coefficient yields a measure of the electron temperature $T_{e}$. Furthermore, if the electron temperature is known, the plasma density could be determined using the bremsstrahlung emission coefficient (Boyd \& Sanderson 2003).

In addition, cyclotron/synchrotron emission would also be expected and would allow the ambient magnetic flux density to be diagnosed. Superimposed on the continuum plasma emission would be emission and absorption lines from the plasma ion species (e.g., $\mathrm{He}, \mathrm{Mg}, \mathrm{Fe}, \mathrm{Na}$ or $\mathrm{H}_{2}, \mathrm{CO}, \mathrm{H}_{2} \mathrm{O}, \mathrm{N}_{2}$ ) constituting the atmospheric plasma, the identification of which would allow the characterization of the plasma species. Furthermore, the electron bombardment of the neutral species populating the atmosphere (as part of the Alfvén ionization process) may 
excite the neutrals that then relax, producing emission that could be identified in the spectra, e.g., $\mathrm{H}_{2}, \mathrm{H}_{2} \mathrm{O}, \mathrm{CO}$, etc. This excitation may include rotational and vibrational excitation, resulting in discrete (and potentially time-dependent) emission lines.

The occurrence of Alfvén ionization is not just restricted to the atmospheres of low-mass objects and may be applicable in other astrophysical environments. In principle, Alfvén ionization can occur in any astrophysical system where there is a magnetized seed plasma and a neutral component that moves relative to the plasma exceeding the critical speed. For example, protoplanetary disks (Woitke et al. 2009) are composed of gas-plasma mixtures that have significant rotational flow speeds due to the gravitational accretion onto the central star. Photoionization is thought to be the main contributor to the plasma, but Alfvén ionization could significantly enhance the plasma density and contribute to the generation of the stellar systems' fossil magnetic fields. Alfvén ionization could also be at work in the atmospheres of other stellar types such as a G-type star (e.g., the Sun), where flow speeds from emerging magnetic flux (i.e., a sunspot or a starspot) could exceed the critical speed, resulting in ionization and enhancing the population of certain ions (Diver et al. 2005).

The authors are grateful to the anonymous referee for constructive comments and suggestions that have improved this paper. Ch.H., C.R.S., and P.B.R. are grateful for the financial support of the European Community under the FP7 by an ERC starting grant. D.A.D. is grateful for funding from the UK Science and Technology Funding Council via grant No. ST/I001808/1.

\section{APPENDIX A \\ COSMIC-RAY IONIZATION OF SUBSTELLAR ATMOSPHERES}

Cosmic ray bombardment of a substellar atmosphere will increase $n_{e}$ via impact ionization of the initially neutral atmosphere. Figure 5 shows the resulting electron number density $n_{e}$ from the bombardment of a brown dwarf atmosphere $([\mathrm{M} / \mathrm{H}]=0.0)$ by cosmic rays. The plot shows that high in the atmosphere ( $p_{\text {gas }} \approx 10^{-4}$ bar), cosmic ray ionization processes can increase the ambient electron number density to $n_{e} \approx 10^{4} \mathrm{~cm}^{-3}$. In the calculation exhibited in Figure 5, the cosmic ray propagation is determined using a 1D Monte Carlo model involving 10,000 cosmic rays that are each binned to different energies according to the spectrum proposed by Nath \& Biermann (1994). Each cosmic ray is assigned a random number in a uniform distribution between zero and one. The cosmic rays then propagate through a column of atmospheric gas $\Delta N$. The random number assigned to the cosmic ray is compared to the cosmic-ray "optical depth" $\sigma(E) \Delta N$, where $\sigma(E)$ is the cross-section for an ionizing collision between a proton and a hydrogen atom (Padovani et al. 2009). If the random number is $<\sigma(E) \Delta N$, then the cosmic ray ionizes the hydrogen atom and loses a given amount of energy $\Delta W(E)$ (Rimmer et al. 2012). The value of $\Delta N$ is always chosen such that $\sigma(E) \Delta N<1$. This process is repeated until the entire atmosphere is traversed. At each step, energy loss due to Alfvén waves generated by the cosmic ray anisotropy is accounted for, as discussed in Skilling \& Strong (1976). This effect is the only magnetic field effect considered.

\section{APPENDIX B}

\section{ATMOSPHERIC FLOWS}

Balanced flows are an idealized view of atmospheric motion, but these flows give a good approximation of the dynamical behavior and expected characteristic flow speeds. In this appendix, we consider a simple fluid dynamical model to describe three standard atmospheric balanced flows: geostrophic, cyclostrophic, and gradient flows. In the subsequent analysis, we follow the texts of Vallis (2008), Holton (2004), SánchezLavega (2011), and Jacobson (2005). Note that in the presence of significant ionization, atmospheres will be composed of a gas-plasma mixture and the dynamical evolution of the system will be affected by electromagnetic fields. The onward evolution of the gas-plasma mixture would be much more complex since the two fluids (plasma and neutral gas) could be collisionally coupled (Diver et al. 2006). In this scenario, the plasma could be described by magnetohydrodynamics (MHD; see Boyd \& Sanderson 2003 for the governing equations), encapsulating the effect of the magnetic field on the charged species. In the low-frequency, MHD regime, the plasma can support a variety of plasma wave and flow phenomena (e.g., Diver et al. 2006; Zaqarashvili et al. 2007; Nakariakov \& Verwichte 2005). Here, we consider an atmosphere predominantly composed of a neutral gas such that the dynamical effect of the plasma is a second order effect.

In a rotating reference frame, the atmospheric gas dynamics are described by

$$
\frac{\partial \rho_{\mathrm{gas}}}{\partial t}+\nabla \cdot\left(\rho_{\mathrm{gas}} \mathbf{u}\right)=0
$$

$$
\begin{gathered}
\rho_{\mathrm{gas}} \frac{D \mathbf{u}}{D t}=-\nabla p_{\mathrm{gas}}-2 \rho_{\mathrm{gas}} \mathbf{\Omega} \times \mathbf{u}+\rho_{\mathrm{gas}} \hat{\mathbf{g}}, \\
\frac{D p_{\mathrm{gas}}}{D t}=-\gamma p_{\mathrm{gas}} \nabla \cdot \mathbf{u}+S, \\
p_{\mathrm{gas}}=h\left(T_{\mathrm{gas}}, \rho_{\mathrm{gas}}\right),
\end{gathered}
$$

where

$$
\hat{\mathbf{g}}=\mathbf{g}-\boldsymbol{\Omega} \times(\boldsymbol{\Omega} \times \mathbf{r})
$$

is the effective gravitational acceleration, $\gamma=5 / 3$ is the ratio of specific heats, and $h$ is the equation of state. The momentum equation includes the pressure gradient force $\left(-\nabla p_{\text {gas }}\right)$, the Coriolis effect $\left(-2 \rho_{\text {gas }} \boldsymbol{\Omega} \times \mathbf{u}\right)$, the centrifugal effect $\left(-\rho_{\text {gas }} \boldsymbol{\Omega} \times\right.$ $(\boldsymbol{\Omega} \times r))$, and gravity $\left(\rho_{\text {gas }} \mathbf{g}\right)$. The energy equation includes an energy source and sink term $S$, describing the rate of heating or cooling of the gas. The temptation is to cast these equations in spherical coordinates with a radial component defined by the gravity g. However, the presence of a centrifugal effect complicates matters and so it is convenient to define the radial direction instead by $\hat{\mathbf{g}}$, encapsulating the centrifugal effect with gravity. Strictly speaking, the surfaces defined by the normal $\hat{\mathbf{g}}$ are not spherical but spheroidal, but for simplicity will be treated as spherical. As a result, the horizontal component of effective gravity is zero, thus simplifying the governing equations. Additionally, further simplification can be sought by focusing on phenomena on a length scale where the sphericity of our rotating body is negligible. In this case, instead of solving the equations in spherical coordinates, we can use a local Cartesian tangent plane on the surface of the sphere (known as the $f$-plane). 
Consider a location with latitude $\phi$ defined on a surface with normal $\hat{\mathbf{g}}$ rotating around the north-south axis. A local Cartesian coordinate system can be set up with the $x$-axis horizontally due east, the $y$-axis horizontally due west, and the $z$-axis vertically upward. The angular velocity can be written as $\boldsymbol{\Omega}=\omega(0, \cos \phi, \sin \phi)$; therefore, the governing dynamical equations become

$$
\begin{aligned}
& \frac{D u_{x}}{D t}=-\frac{1}{\rho_{\mathrm{gas}}} \frac{\partial p_{\mathrm{gas}}}{\partial x}+f u_{y}, \\
& \frac{D u_{y}}{D t}=-\frac{1}{\rho_{\mathrm{gas}}} \frac{\partial p_{\mathrm{gas}}}{\partial y}-f u_{x}, \\
& \frac{D u_{z}}{D t}=-\frac{1}{\rho_{\mathrm{gas}}} \frac{\partial p_{\mathrm{gas}}}{\partial z}+\hat{g},
\end{aligned}
$$

where $f=2 \omega \sin \phi$ is the Coriolis parameter characterizing the significance of the Coriolis effect as a function of position on the surface. If we assume that the vertical velocity component is negligible and that the vertical component of the Coriolis effect is small in comparison with the effective gravity, we can restrict our attention to a horizontal plane (i.e., $u_{z}=0$ ). These approximations are the hydrostatic and traditional approximations, respectively, yielding the so-called "primitive" equations of motion.

\section{B.1. Geostrophic Flow}

The Rossby number is defined as

$$
R o=\frac{|(\mathbf{u} \cdot \nabla) \mathbf{u}|}{|\mathbf{\Omega} \times \mathbf{u}|}=\frac{U}{L f},
$$

characterizing the significance of the Coriolis effect, hence rotation on the system. Ro $\ll 1$ defines a system strongly affected by the Coriolis effect and $R o \gg 1$ defines a system where inertial effects dominate. In the steady state $(\partial / \partial t=0)$, if the local Rossby number is small $(R o \ll 1)$, then a geostrophic wind balance is achieved:

$$
\begin{aligned}
& u_{x}=-\frac{1}{f \rho_{\mathrm{gas}}} \frac{\partial p_{\mathrm{gas}}}{\partial y} \\
& u_{y}=\frac{1}{f \rho_{\mathrm{gas}}} \frac{\partial p_{\mathrm{gas}}}{\partial x} .
\end{aligned}
$$

Geostrophic wind balance results from the balance between the Coriolis effect and the pressure gradient force. Note that geostrophic flows are invalid at the equator because $f=0$.

\section{B.2. Cyclostrophic Flow}

Transforming from Cartesian to cylindrical polar coordinates, the primitive equations of atmospheric motion in the $f$-plane become

$$
\begin{aligned}
& \rho_{\text {gas }} \mathcal{D} u_{r}=-\frac{\partial p_{\text {gas }}}{\partial r}+\rho_{\text {gas }} f+\rho_{\text {gas }} \frac{u_{\theta}^{2}}{r} \\
& \rho_{\text {gas }} \mathcal{D} u_{\theta}=-\frac{1}{r} \frac{\partial p_{\text {gas }}}{\partial \theta}-\rho_{\text {gas }} f-\rho_{\text {gas }} \frac{u_{\theta} u_{r}}{r}, \\
& \rho_{\text {gas }} \mathcal{D} u_{z}=-\frac{\partial p_{\text {gas }}}{\partial z}+\rho_{\text {gas }} \hat{g}
\end{aligned}
$$

where

$$
\mathcal{D}=\frac{\partial}{\partial t}+u_{r} \frac{\partial}{\partial r}+\frac{u_{\theta}}{r} \frac{\partial}{\partial \theta}+u_{z} \frac{\partial}{\partial z} .
$$

The latter terms in the $r$ and $\theta$ components of the momentum equation (Equations (B12) and (B13), respectively) correspond to the local centrifugal $\left(u_{\theta}^{2} / r\right)$ and local Coriolis effects $\left(u_{\theta} u_{r} / r\right)$, as experienced in the non-inertial frame of a rotating fluid parcel of atmospheric gas. Cyclostrophic flows are characterized by the balance between the pressure gradient force and the centrifugal effect. This characterization is applicable when the horizontal length scale under consideration is small enough such that the global Coriolis effect $\left(\rho_{\text {gas }} f\right)$ can be neglected. In the steady state, assuming the hydrostatic approximation and uniform circular motion $\left(u_{r}=0, \partial p_{\text {gas }} / \partial \theta=0\right)$ where the centripetal force is provided by the pressure gradient force, the cyclostrophic approximation is

$$
\frac{\rho_{\mathrm{gas}} u_{\theta}}{r}=\frac{\partial p_{\mathrm{gas}}}{\partial r}
$$

yielding a cyclostrophic wind speed,

$$
u_{\theta}=\left(\frac{r}{\rho_{\mathrm{gas}}} \frac{\partial p_{\mathrm{gas}}}{\partial r}\right)^{1 / 2} .
$$

\section{B.3. Gradient Flow}

Retaining the primitive equations in a local cylindrical geometry, another force balance can be obtained between the global Coriolis effect, the pressure gradient force, and the local centrifugal effect. For simplicity, uniform circular motion is assumed $\left(u_{r}=0\right)$, the hydrostatic approximation is assumed, and $\partial p_{\text {gas }} / \partial \theta=0$. These assumptions yield

$$
-\frac{\rho_{\mathrm{gas}} u_{\theta}^{2}}{r}=-\frac{\partial p_{\mathrm{gas}}}{\partial r}+\rho_{\mathrm{gas}} f u_{\theta} .
$$

Solving the quadratic equation, the gradient flow speed is

$$
u_{\theta}=-\frac{1}{2} r f \pm \frac{1}{2} r\left(f^{2}+\frac{4}{r \rho_{\mathrm{gas}}} \frac{\partial p_{\mathrm{gas}}}{\partial r}\right)^{1 / 2}
$$

\section{REFERENCES}

Alfvén, H. 1960, RvMP, 32, 710

Antonova, A., Doyle, J. G., Hallinan, G., Bourke, S., \& Golden, A. 2008, A\&A, 487,317

Antonova, A., Doyle, J. G., Hallinan, G., Golden, A., \& Koen, C. 2007, A\&A, 472,257

Antonova, A., Hallinan, G., Doyle, J. G., et al. 2013, A\&A, 549, A131

Becker, K. H., Schoenbach, K. H., \& Eden, J. G. 2006, JPhD, 39, R55

Beebe, R. 1997, Jupiter the Giant Planet (2nd ed.; Washington, DC: Smithsonian Institution Press)

Berger, E. 2006, ApJ, 648, 629

Berger, E., Rutledge, R. E., Phan-Bao, N., et al. 2009, ApJ, 695, 310

Borrero, J. M., Lites, B. W., \& Solanki, S. K. 2008, A\&A, 481, L13

Boyd, T. J. M., \& Sanderson, J. J. 2003, The Physics of Plasmas (Cambridge: Cambridge Univ. Press)

Bransden, B. H., \& Joachin, C. J. 2000, Quantum Mechanics (2nd ed.; Reading, MA: Addison-Wesley)

Brenning, N. 1992, SSRv, 59, 209

Chang, Z. S., Zhao, N., \& Yuan, P. 2010, PhPl, 17, 113514

Choi, D. S., Showman, A. P., \& Vasavada, A. R. 2010, Icar, 207, 359

Christensen, U. R., Holzwarth, V., \& Reiners, A. 2009, Natur, 457, 168

Cooper, C. S., \& Showman, A. P. 2005, ApJL, 629, L45

Danielsson, L. 1973, Ap\&SS, 24, 459

Dehn, M. 2007, PhD thesis, Univ. Hamburg 
Diver, D. A. 2013, A Plasma Formulary for Physics, Technology, and Astrophysics (2nd ed.; Berlin: Wiley-VCH)

Diver, D. A., Fletcher, L., \& Potts, H. E. 2005, SoPh, 227, 207

Diver, D. A., Potts, H. E., \& Teodoro, L. F. A. 2006, NJoP, 8, 265

Dobbs-Dixon, I., Agol, E., \& Burrows, A. 2012, ApJ, 751, 87

Dobbs-Dixon, I., Cumming, A., \& Lin, D. N. C. 2010, ApJ, 710, 1395

Dobbs-Dixon, I., \& Lin, D. N. C. 2008, ApJ, 673, 513

Donati, J.-F., \& Landstreet, J. D. 2009, ARA\&A, 47, 333

Dunning, F. B., \& Hulet, R. G. 1996, Atomic, Molecular, and Optical Physics: Atoms and Molecules, Vol. 29b (London: Academic Press)

Fahleson, U. V. 1961, PhFl, 4, 123

Fridman, A. 2008, Plasma Chemistry (1st ed.; New York: Cambridge Univ. Press)

Guo, Y., Yuan, P., Shen, X., \& Wang, J. 2009, PhyS, 80, 035901

Hallinan, G., Antonova, A., Doyle, J. G., et al. 2006, ApJ, 653, 690

Hallinan, G., Antonova, A., Doyle, J. G., et al. 2008, ApJ, 684, 644

Hallinan, G., Bourke, S., Lane, C., et al. 2007, ApJL, 663, L25

Hammel, H. B., Rages, K., Lockwood, G. W., Karkoschka, E., \& de Peter, I. 2001, Icar, 153, 229

Hartman, H., Schef, P., Lundin, P., et al. 2005, MNRAS, 361, 206

Helling, Ch., Ackerman, A., Allard, F., et al. 2008a, MNRAS, 391, 1854

Helling, Ch., Jardine, M., \& Mokler, F. 2011a, ApJ, 737, 38

Helling, Ch., Jardine, M., Stark, C. R., \& Diver, D. A. 2013, ApJ, 767, 136

Helling, Ch., Jardine, M., Witte, S., \& Diver, D. A. 2011b, ApJ, 727, 4

Helling, Ch., Woitke, P., \& Thi, W.-F. 2008b, A\&A, 485, 547

Heng, K. 2012, ApJL, 761, L1

Heng, K., Menou, K., \& Phillips, P. J. 2011, MNRAS, 413, 2380

Holton, J. R. 2004, An Introduction to Dynamic Meteorology (4th ed.; Burlington, MA: Elsevier Academic Press)

Jacobson, M. Z. 2005, Fundamentals of Atmospheric Modeling (2nd ed.; Cambridge: Cambridge Univ. Press)

Joergens, V., Fernández, M., Carpenter, J. M., \& Neuhäuser, R. 2003, ApJ, 594,971

Khodachenko, M. L., Alexeev, I., Belenkaya, E., et al. 2012, ApJ, 744, 70

Lai, S. T. 2001, RvGeo, 39, 471

Lewis, N. K., Showman, A. P., Fortney, J. J., Marley, M. S., \& Freedman, R. S. 2010, ApJ, 720, 344

Li, C., Ebert, U., \& Hundsdorfer, W. 2009, JPhD, 42, 202003

Machida, S., \& Goertz, C. K. 1986, JGRA, 91, 11965

MacLachlan, C. S., Diver, D. A., \& Potts, H. E. 2009, NJPh, 11, 063001

MacLachlan, C. S., Potts, H. E., \& Diver, D. A. 2013, PSST, 22, 015025

McBride, J. B., Ott, E., Boris, J. P., \& Orens, J. H. 1972, PhFl, 15, 2367

McLean, M., Berger, E., Irwin, J., Forbrich, J., \& Reiners, A. 2011, ApJ, 741, 27

McLean, M., Berger, E., \& Reiners, A. 2012, ApJ, 746, 23

McNeil, W. J., Lai, S. T., \& Murad, E. 1990, JGRA, 95, 10345

Menou, K., \& Rauscher, E. 2009, ApJ, 700, 887
Nakariakov, V. M., \& Verwichte, E. 2005, LRSP, 2, 3

Nath, B. B., \& Biermann, P. L. 1994, MNRAS, 267, 447

Newell, P. T. 1985, RvGeo, 23, 93

Nichols, J. D., Nurleigh, M. R., Caswell, S. L., et al. 2012, ApJ, 760, 59

Osten, R. A., Phan-Bao, N., Hawley, S. L., Neill Reid, I., \& Ojha, R. 2009, ApJ, 700, 1750

Padovani, M., Galli, D., \& Glassgold, A. E. 2009, A\&A, 501, 619

Person, J. C., Resendes, D., Petschek, H., \& Hastings, D. E. 1990, JGRA, 95 , 4039

Porco, C. C., Baker, E., Barbara, J., et al. 2005, Sci, 307, 1243

Priest, E. R. (ed.) 1985, Solar System Magnetic Fields (1st ed.; Dordrecht: Reidel)

Rauscher, E., \& Menou, K. 2010, ApJ, 714, 1334

Reiners, A. 2012, LRSP, 8, 1

Reiners, A., Joshi, N., \& Goldman, B. 2012, ApJ, 143, 93

Rimmer, P. B., \& Helling, Ch. 2013, ApJ, 774, 108

Rimmer, P. B., Herbst, E., Morata, E., \& Roueff, E. 2012, A\&A, 537, A7

Route, M., \& Wolszczan, A. 2012, ApJL, 747, L22

Sánchez-Lavega, A. 2001, A\&A, 377, 354

Sánchez-Lavega, A. 2004, ApJL, 609, L87

Sánchez-Lavega, A. 2011, An Introduction to Planetary Atmospheres (1st ed.; Boca Raton, FL: Taylor and Francis)

Scholz, A., \& Eislöffel, J. 2005, A\&A, 429, 1007

Showman, A. P., Cooper, C. S., Fortney, J. J., \& Marley, M. S. 2008, ApJ, 682,559

Showman, A. P., Fortney, J. J., Lian, Y., et al. 2009, ApJ, 699, 564

Showman, A. P., \& Guilot, T. 2002, A\&A, 385, 166

Shulyak, D., Seifahrt, A., Reiners, A., Kochukhov, O., \& Piskunov, N. 2011, MNRAS, 418, 2548

Skilling, J., \& Strong, A. W. 1976, A\&A, 53, 253

Sromovsky, L. A., \& Fry, P. M. 2005, Icar, 179, 459

Stark, C. R., Potts, H. E., \& Diver, D. A. 2006, A\&A, 457, 365

Uman, M. A., \& Orville, R. E. 1964, JGR, 69, 5151

Vallis, G. V. 2008, Atmospheric and Oceanic Fluid Dynamics Fundamentals and Large-Scale Circulation (3rd ed.; Cambridge: Cambridge Univ. Press)

Vidotto, A. A., Jardine, M., \& Helling, Ch. 2011a, MNRAS, 411, L46

Vidotto, A. A., Jardine, M., \& Helling, Ch. 2011b, MNRAS, 414, 1573

Witte, S., Helling, Ch., Barman, T., Heidrich, N., \& Hauschildt, P. H. 2011, A\&A, 529, A44

Woitke, P., \& Helling, Ch. 2003, A\&A, 399, 297

Woitke, P., Kamp, I., \& Thi, W.-F. 2009, A\&A, 501, 383

Yu, S., Doyle, J. G., Kuznetsov, A., et al. 2012, ApJ, 752, 60

Zapatero Osorio, M. R., Martín, E. L., Bouy, H., Tata, R., \& Deshpande, R. 2006, ApJ, 647, 1405

Zaqarashvili, T. V., Oliver, R., Ballester, J. L., \& Shergelashvili, B. M. 2007, A\&A, 470, 815

Zethson, T., Gull, T. R., Hartman, H., et al. 2001, AJ, 122, 322 